\title{
Optical characterization of multi-scale morphologically complex heterogeneous media - Application to snow with soot impurities
}

\author{
Xiaoyu Dai, Sophia Haussener* \\ Laboratory of Renewable Energy Science and Engineering, EPFL, Station 9, Lausanne 1015, Switzerland
}

\section{A R T I C L E I N F O}

\section{Article history:}

Received 22 August 2017

Revised 28 November 2017

Accepted 30 November 2017

Available online 7 December 2017

\begin{abstract}
A B S T R A C T
A multi-scale methodology for the radiative transfer analysis of heterogeneous media composed of morphologically-complex components on two distinct scales is presented. The methodology incorporates the exact morphology at the various scales and utilizes volume-averaging approaches with the corresponding effective properties to couple the scales. At the continuum level, the volume-averaged coupled radiative transfer equations are solved utilizing $(i)$ effective radiative transport properties obtained by direct Monte Carlo simulations at the pore level, and (ii) averaged bulk material properties obtained at particle level by Lorenz-Mie theory or discrete dipole approximation calculations. This model is applied to a soot-contaminated snow layer, and is experimentally validated with reflectance measurements of such layers. A quantitative and decoupled understanding of the morphological effect on the radiative transport is achieved, and a significant influence of the dual-scale morphology on the macroscopic optical behavior is observed. Our results show that with a small amount of soot particles, of the order of $1 \mathrm{ppb}$ in volume fraction, the reduction in reflectance of a snow layer with large ice grains can reach up to $77 \%$ (at a wavelength of $0.3 \mu \mathrm{m}$ ). Soot impurities modeled as compact agglomerates yield 2-3\% lower reduction of the reflectance in a thick show layer compared to snow with soot impurities modeled as chain-like agglomerates. Soot impurities modeled as equivalent spherical particles underestimate the reflectance reduction by $2-8 \%$. This study implies that the morphology of the heterogeneities in a media significantly affects the macroscopic optical behavior and, specifically for the soot-contaminated snow, indicates the non-negligible role of soot on the absorption behavior of snow layers. It can be equally used in technical applications for the assessment and optimization of optical performance in multi-scale media.
\end{abstract}

(c) 2017 Elsevier Ltd. All rights reserved.

\section{Introduction}

Radiative transfer in heterogeneous media is observed in a variety of significant technical applications. These media serve as hightemperature solar absorbers in solar energy conversion processes [1], as solar absorber and charge generators in photovoltaics devices [2], or reflect the solar radiation back into the atmosphere from the snow covered earth surface [3,4]. The maximization of the absorption in solar energy conversion devices or the accurate characterization of the reflection behavior of earth surfaces for climate modeling are objectives when investigating these systems. The radiative characterization of such media is challenging and is generally done by a combined two-step approach $[5,6]$ : $(i)$ determination of the radiative properties on the scale of the typical structural heterogeneity using Mie theory or Monte Carlo methods, and (ii) solution of the single-phase radiative transfer equation

\footnotetext{
* Corresponding author.

E-mail address: sophia.haussener@epfl.ch (S. Haussener).
}

(RTE) using radiative transfer theory for the determination of the macroscopic optical properties. This approach relies on the validity of the phenomenological radiative transfer theory on the macroscale [7] and on the accurate prediction of the radiative properties. A variety of systems have been investigated using this approach and few examples studies include the characterization of packed beds of large opaque and semi-transparent spheres [8,9], or foams and fibrous media [10]. Mie theory and its modification are typically applicable to structures with spherical or fibrous shapes only. To account for more complex morphologies, the direct solution of the Maxwell's equations is required. Examples of the solution of the Maxwell's equation or its approximation by discrete dipole approaches have been presented for three-dimensionally ordered ceria particles [11,12]. For larger-scales down to about a micrometer, microscopy-based approaches (e.g. tomography) are used in order to incorporate the detailed morphological information. Monte Carlo techniques are then applied in order to determine the radiative properties in a spectral range for which geometrical optics can be applied [13-15]. Alternatively, combined experimentalnumerical approaches can be used for the determination of the 


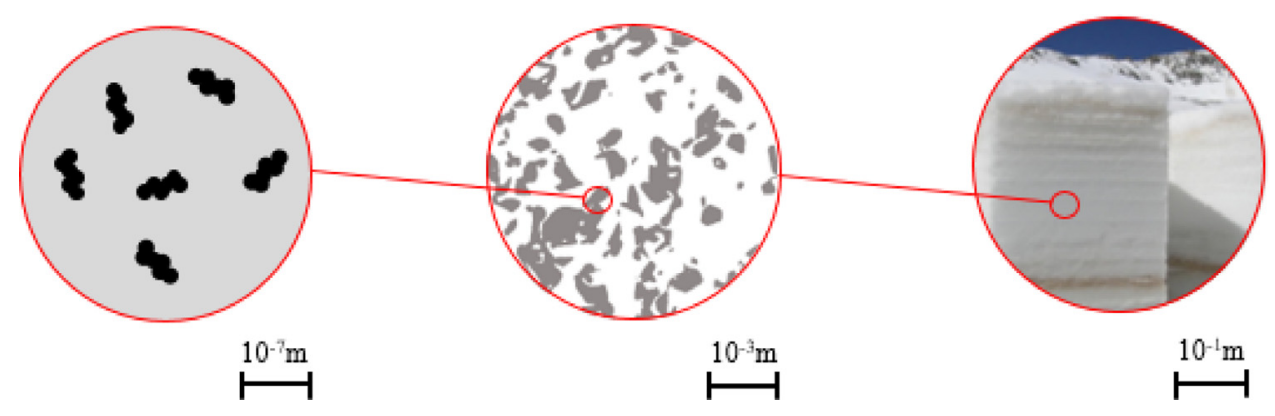

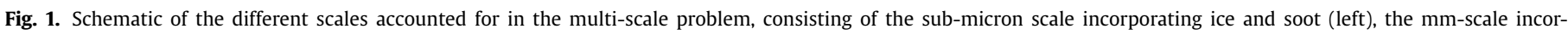
porating air and ice-soot phases (middle), and the macro-scale incorporating the semi-transparent snow pack (right).

radiative properties: the macroscopic optical properties are measured and computational techniques are used to inversely estimate the radiative properties [5]. For example, the radiative properties of a packed bed of zinc-oxide have been determined using directional transmittance measurements in combination with Monte Carlo simulations [16], or the transport radiative properties of porous ceria have been determined using directional-hemispherical reflectance and directional transmittance measurements in combination with two-flux approximation and Monte Carlo calculations $[17,18]$.

An additional complexity is added to radiative transport problems in heterogeneous media when the media exhibit morphological details at various scales, namely structural details with large characteristic size parameters $(x=\pi \cdot d / \lambda>1, d$ being a characteristic length) and structural details with small characteristic size parameters $(x<1)$. A variety of technical and naturally occurring systems exhibit such morphologies, e.g. photoelectrodes synthesized using host-guest approaches [19], solar absorbers with dual-scale porosities [20], or snow packs with soot impurities [21]. In order to optimize the multi-scale morphologies for better absorption behavior (e.g. of the solar absorber) or to increase the understanding of the reflection behavior as a result of these multi-scale morphologies (e.g. for the snow pack with soot impurities) theoretical approaches which can accurately account for the complex morphologies at the various scales are required. Such approaches not only provide in-depth understanding of the morphological influence on the macroscopic optical characteristics, but they also help to de-convolute the influence associated to the bulk material properties, multi-scale morphologies, and boundary conditions on the macroscopic radiative behavior. Furthermore, they provide pathways for morphology tailoring and materials-by-design on multiple scales for improved application performance.

We provide an advancement of the general theoretical radiative characterization approach to heterogeneous media, considering media with morphological characteristic at two distinct scales described by their size parameters significantly above and below one. We apply the method to an example application: sootcontaminated snow layers.

\section{Problem statement and assumptions}

The multi-scale media considered exhibits morphological characteristics at two distinct scales, where the small scale is characterized by a small size parameter (i.e. diameter/wavelength), $x<1$, and the medium scale is characterized by $x>1$. The different scales are schematically shown in Fig. 1 for our application example: a snow pack with agglomerated soot impurities embedded in the ice particles. The snow layer (the macro-scale) is composed of two phases (medium-scale): one transparent phase (air) and one semitransparent phase (ice with soot impurities modeled as one homogeneous ice-soot phase). The small-scale investigations look into the details of the complex soot agglomerates embedded in the ice (submicron-scale).

The simplest case of such a medium consists of three components: air, ice, and soot. The physical properties of each of these components are assumed (i) isotropic, (ii) homogeneous, (iii) nonpolarizing and polarization-independent, and (iv) independent of the radiation intensity and temperature. The components are at local thermodynamic equilibrium, and at rest compared to the speed of light and therefore quasi-steady radiative transport is assumed.

The physical properties - the complex index of refraction - of the component with characteristic sizes at the sub-micron scale are assumed to be size independent and continuous. The components are randomly oriented in the host component and the clearance between the components is large enough that independent scattering can be assumed.

The component with characteristic sizes at the mm-scale are assumed to be much larger than the typical wavelengths of interest and, consequently, the laws of geometrical optics are valid. Diffraction effects and dependent scattering effects are neglected. The two-phase media at the mm-scale is assumed to be composed of a transparent phase (air) and a semi-transparent, homogeneous and isotropic phase (ice-soot mixture). The snow layer is exposed to external radiation (solar irradiation) of either collimated or diffuse character.

\section{Methodology}

The methodology accounts for three scales described as small $(x<1)$, medium $(x>1)$, and large scale $(x \gg 1)$, and consists of the following steps: $(i)$ obtaining the detailed 3D morphology of the sub-micron scaled structures $(x<1)$ and its incorporation into the solution of the Maxwell's equations for the determination of the spectrally-resolved discrete-scale absorption and scattering efficiency factors and scattering phase functions, (ii) obtaining the detailed 3D morphology of the micron sized structures $(x>1)$ and its incorporation into the solution of the discretescale radiative transfer equations (RTEs) for the determination of the spectrally-resolved effective extinction and scattering coefficients and effective scattering phase functions, and (iii) incorporation of the two-scale effective radiative transport properties into the coupled multi-phase volume-averaged RTEs for the determination of the spectrally-resolved averaged intensity vector field and heat transfer fluxes for the determination of reflectance, absorptance, and transmittance. Fig. 2 shows the flowchart of the coupling between the effective properties at distinct scales and the macroscopic optical behavior.

\subsection{Small-scale investigations}

The bulk properties account for morphological characteristics at the sub-micron scale $(x<1)$. Two approaches are chosen for 


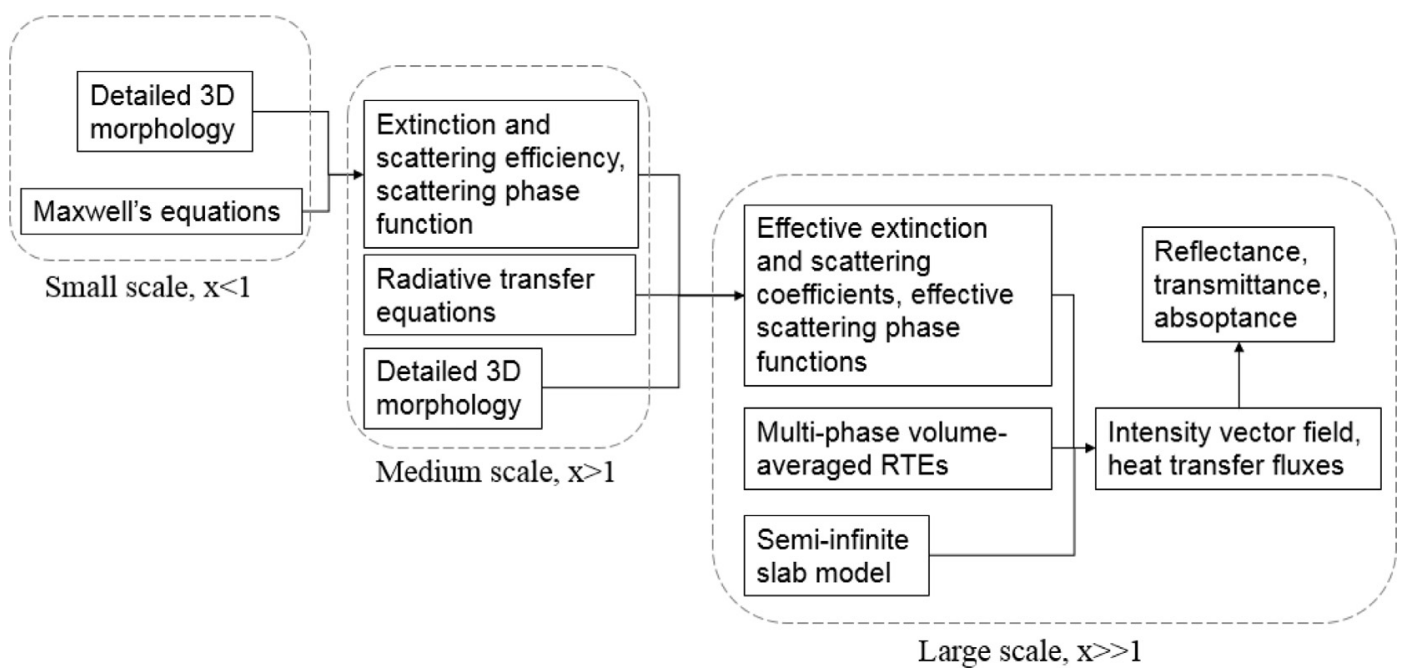

Fig. 2. Flow diagram of the analysis path of the multi-scale problem consisting of the small, medium, and large scales.

the determination of the sub-micron scale radiative properties: (i) Lorenz-Mie theory for spherically shaped objects, and (ii) the direct solution of the Maxwell equation on non-spherical objects [22]. The Maxwell's equations are solved by using the discrete dipole approximation (DDA) where the complex structures are approximated by a large numbers of dipoles and the calculated dipole moments are used for the determination of the scattering and extinction efficiency coefficient factors, and scattering phase function $[23,24]$.

Lorenz-Mie theory is generally used to solve the electromagnetic field around an irradiated perfectly spherical particle. It provides the exact solutions to Maxwell's equations with the corresponding boundary conditions [22]. In the case when the spheres are surrounded by a highly absorbing medium, a generalized form of the Lorenz-Mie theory can be used [25]. We used the open source code BHMIE for the calculations for the Lorenz-Mie calculations [22] and an open source code provided by Jeppe et al. for the generalized Lorenz-Mie calculations [25].

However, when the particles have arbitrary and complex structures, such as being clustered or aggregated, Lorenz-Mie theory is not applicable, instead the electromagnetic field around these complex structures has to be directly determined solving the Maxwell's equations with this complex interface boundary condition. We used the discrete dipole approximation (DDA) model, introduced by Purcell and Pennypacker [26], to solve the Maxwell's equation in this complex computational domain, as the direct solution is computationally demanding. Wheeler et al. [11] compared the direct solution of the Maxwell's equations with the DDA approach for a complex particle and suggested that DDA can provide accurate solutions at reduced computational cost. This observation coincides with our experience.

We used DDSCAT, an open source code for DDA calculations, which solves the problem of a finite target interacting with a monochromatic plane wave incident from infinity, for the calculation of the radiation characteristics of more realistic morphologies of the soot agglomerates [23,27]. The applicability of DDA is given only if a specific prerequisite is satisfied: the discrete lattice spacing should be small enough compared to the structure and the radiation wavelength. A numerical condition can be formulated based on this prerequisite, namely the system needs to satisfy $|m| \omega s \leq 1$ ( $m$ being the refractive index, $\omega=2 \pi / \lambda, s$ being the lattice spacing satisfying $V=N s^{3}, N$ being the number of dipoles). It is known that for a material with a large refractive index, $|m| \gg 1$, DDA can significantly overestimate absorption cross sections, even when respecting the condition $|m| \omega s \leq 1$ [23,27-29]. However, this case is not relevant in our application. We perform the DDA calculation for targets in multiple random orientations, to obtain orientation-independent optical properties.

The characterizations of the radiative properties at the smallscale are coupled to the medium-scale by summing the extinction coefficient of the independently absorbing and scattering agglomerates (determined by Mie or DDA calculations),

$\beta_{\mathrm{d}, 2 b, \lambda}=\frac{3}{2} \frac{f_{v}}{a_{\mathrm{eff}}}\left(Q_{\mathrm{a}, \lambda}+Q_{\mathrm{s}, \lambda}\right)=\kappa_{\mathrm{d}, 2 b, \lambda}+\sigma_{\mathrm{d}, 2 b, \lambda}$,

for which $f_{\mathrm{v}}$ is the volume fraction in the host medium, and $a_{\mathrm{eff}}$ is the effective radius of the equivalent-volume sphere [30].

The absorbing behavior of the embedding phase (phase $a$ is homogeneous ice, phase $b$ is the agglomerates) is given by electromagnetic theory [22],

$\kappa_{\mathrm{d}, 2 a, \lambda}=\frac{4 \pi k_{2 a, \lambda}}{\lambda}$,

and allows for the determination of the extinction coefficient of the ice-soot mixture phase (phase 2) [30],

$\beta_{\mathrm{d}, 2, \lambda}=\left(1-f_{v}\right) \kappa_{\mathrm{d}, 2 a, \lambda}+\beta_{\mathrm{d}, 2 b, \lambda}$,

to be used in subsequent medium-scale calculations of the effective properties. The scattering function is directly applicable to the medium-scale calculations.

\subsection{Medium-scale investigations}

Volume-averaging approaches provide the theoretical basis for the radiative characterization in morphologically-complex multiphase media, introducing effective transport properties to account for the geometrical complexity. These effective properties can be postulated and defined by volume averaging of the two radiative transfer equations (RTEs) of the two discrete-scale phases (in our example air and ice-soot mixture) and by applying the proper inter-phase boundary conditions. The utilization of the spatial averaging theorem and subsequent closure of the resulting equations leads to the introduction of the effective properties [31,32]. For a system with a two phase medium (phase $i$ and phase $j$ ), the required effective properties are $\sigma_{\mathrm{s}, i i}, \sigma_{\mathrm{s}, i j}, \beta_{i}, \Phi_{i i}$, and $\Phi_{i j} . \sigma_{\mathrm{s}, i i}$ and $\Phi_{i i}$ are the effective scattering coefficient and phase function related to internal scattering and interface reflection phenomena (e.g. $\sigma_{\mathrm{s}, i i}=\sigma_{\mathrm{s}, \mathrm{d}, i}+\sigma_{\mathrm{s}, \text { int }, i i}$, see Eqs. (1) and (2) for $\sigma_{\mathrm{s}, \mathrm{d}, i}$ of phase 
2) within phase $i . \sigma_{i j}$ and $\Phi_{i j}$ are the effective scattering coefficient and phase function associated with radiation refracting from phase $i$ to phase $j$. The effective extinction coefficient $\beta_{i}$ is the sum of effective absorption and scattering coefficients, incorporating morphology-dependent properties $\left(\sigma_{\mathrm{s}, \text { int,ii }}\right.$ and $\left.\sigma_{\mathrm{s}, \text { int,ij }}\right)$ as well as bulk-phase properties ( $\beta_{\mathrm{d}, \text { }}$, see Eq. (3) for phase 2 ). A collisionbased Monte Carlo method is used for the determination of the effective properties [33,34]. A large number of stochastic rays is launched within a representative elementary volume (REV). The rays are emitted isotropically and are uniformly distributed over the REV. They undergo internal scattering and absorption, and reflection and refraction at the phase $i$ - phase $j$ interfaces, where the Fresnel equations and the generalized Snell's law are applied. We assumed the soot particles to be well dispersed within the ice grain, therefore the air-ice/soot mixture interface was treated equivalent to an air-ice interface.

The exact morphology of the morphologically complex media on the medium-scale (mm-scale) is incorporated into the calculations by using X-ray tomography for obtaining the 3D morphology and a histogram-based segmentation and digitalization, following the approach described by Petrasch et al. [35] and Haussener et al. [33]. The calculations are therefore performed on the exact morphology, i.e. the tomographic data, where a level-set based approach is chosen for the identification of the phase interface. This algorithm identifies the intersection point between a ray and the phase interface by following the ray in discrete steps until the boundary is reached, followed by applying the bisection method for the exact determination of the interface. The surface normal is calculated by using a linear interpolation in three dimensions.

\subsection{Large-scale investigations}

On the large-scale (continuum-scale) the intensity vector field within a heterogeneous medium composed of two homogeneous phases is given by two volume-averaged RTEs [31]:

$$
\begin{aligned}
\hat{\mathbf{s}} \cdot \nabla I_{i, \lambda}(\mathbf{x}, \hat{\mathbf{s}})= & -\left[\kappa_{\mathrm{d}, i, \lambda}+\sigma_{\mathrm{s}, \mathrm{d}, i, \lambda}+\sigma_{\mathrm{s}, \text { int }, i i, \lambda}+\sigma_{\mathrm{s}, \text { int }, i j, \lambda}\right] I_{i, \lambda}(\mathbf{x}, \hat{\mathbf{s}}) \\
+ & n_{i, \lambda}^{2} \kappa_{\mathrm{d}, i, \lambda} I_{\mathrm{b}, i, \lambda}(\mathbf{x}) \\
+ & \frac{1}{4 \pi} \int_{4 \pi} I_{i, \lambda}\left(\mathbf{x}, \hat{\mathbf{s}}_{\mathrm{in}}\right)\left[\sigma_{\mathrm{s}, \mathrm{d}, i, \lambda} \Phi_{\mathrm{d}, i, \lambda}\left(\hat{\mathbf{s}}_{\mathrm{in}}, \hat{\mathbf{s}}\right)\right. \\
+ & \left.\sigma_{\mathrm{s}, \text { int }, i i, \lambda} \Phi_{\text {int }, i i, \lambda}\left(\hat{\mathbf{s}}_{\mathrm{in}}, \hat{\mathbf{s}}\right)\right] \mathrm{d} \Omega_{\text {in }} \\
+ & \frac{\sigma_{\mathrm{s}, \text { int }, j i, \lambda}}{4 \pi} \int_{4 \pi} I_{j, \lambda}\left(\mathbf{x}, \hat{\mathbf{s}}_{\text {in }}\right) \Phi_{\text {int }, j i, \lambda}\left(\hat{\mathbf{s}}_{\mathrm{in}}, \hat{\mathbf{s}}\right) \mathrm{d} \Omega_{\text {in }}, \\
& i, j=1,2 \text { and } i \neq j
\end{aligned}
$$

where $I_{i}$ represents the volume-averaged intensity of phase $i ; \kappa$, $\sigma, \beta, \Phi$, represent the effective or discrete absorption coefficient, scattering coefficient, extinction coefficient, and scattering phase function. The subscript $d$ represents bulk properties of the phases which incorporate effects of morphological characteristics with dimensions at the small scale $(x<1)$ and are calculated according to the description in Section 3.1. The subscript int represents the effective properties which incorporate effects of morphological characteristics with dimensions at the medium scale $(x>1)$ and are calculated according to the description in Section 3.2. A path length-based Monte Carlo method is applied to solve the two volume-averaged RTEs, Eq. (4), with appropriate boundary conditions [31]. We use a semi-infinite slab of thickness $l_{\text {slab }}$ as the model domain. If not stated otherwise, the reference thickness is $l_{\text {slab }}=4 \mathrm{~cm}$. The boundary conditions for the slab are: diffuse irradiating flux, $\dot{q}_{\text {in }}$, from the front (at $z=0$ ), and absorbing front and back (at $z=l_{\text {slab }}$ ) boundaries. The lateral edges of the slab are assumed infinitely large, equal to periodic boundaries. The medium considered here is a cold medium. The temperature is so low so that the radiative transfer can be decoupled from other modes of
Table 1

Morphological characteristics of the characteristic snow samples and of snow samples approximated by a packed bed of equivalent spheres (subscript eq) [14].

\begin{tabular}{lllll}
\hline Type & Porosity & $\begin{array}{l}\text { Specific } \\
\text { surface } \\
\left(\mathrm{mm}^{-1}\right)\end{array}$ & $\begin{array}{l}\text { Edge length } \\
\text { of cubical } \\
\text { REV }(\mathrm{mm})\end{array}$ & $\begin{array}{l}\text { Mean ice } \\
\text { particle } \\
\text { diameter }(\mathrm{mm})\end{array}$ \\
\hline ds & \multirow{2}{*}{0.85} & 8.2 & 0.63 & 0.05 \\
ds_eq & & & & 0.10 \\
mII & 0.81 & 5.5 & 1.37 & 0.13 \\
mII_eq & & & & 0.19 \\
dh & 0.67 & 2.8 & 3.33 & 0.40 \\
dh_eq & & & & 0.58 \\
ws & 0.38 & 3.0 & 3.93 & 0.66 \\
ws_eq & & & & 0.73 \\
\hline
\end{tabular}

heat transfer and the temperature and its variation can be ignored [30].

The macroscopic optical properties, namely, the fraction of incident radiation which is reflected, $R$, transmitted, $T r$, and absorbed, $A$, by the slab with thickness $l_{\text {slab }}$ are determined based on the intensity fields:

$R=\frac{\sum_{i=1}^{2} \int_{4 \pi} I_{i, \lambda}^{-}(z=0, \hat{\mathbf{s}}) \mathrm{d} \Omega}{\dot{q}_{\text {in }}}$

$\operatorname{Tr}=\frac{\sum_{i=1}^{2} \int_{4 \pi} I_{i, \lambda}^{+}\left(z=l_{\text {slab }}, \hat{\mathbf{s}}\right) \mathrm{d} \Omega}{\dot{q}_{\text {in }}}$

$A=1-R-T r$

The superscript of $I$ describes the intensities in forward or backward direction $(+,-)$, and the subscript $i$ whether we use the intensity of phase 1 (air) or phase 2 (ice-soot mixture).

\section{Application to snow with soot impurities}

The methodology introduced was applied to snow layers composed of a packed bed of air and ice particles, where the ice particles are contaminated with soot impurities. The importance for the characterization of the radiative behavior of snow is related to the fact that the total net anthropogenic radiative forcing is predicted to be $1.6+0.8 /-1.0 \mathrm{~W} / \mathrm{m}^{2}$, of which in average $0.1 \mathrm{~W} / \mathrm{m}^{2}$ are associated to changes in surface albedo due to soot impurities in snow but with a large uncertainty of $+/-0.1 \mathrm{~W} / \mathrm{m}^{2}$ [36].

\subsection{Agglomerate and snow morphologies}

Snow and agglomerated soot have both very complex and stochastic morphologies. We chose four distinct snow samples (Fig. 3): (i) decomposed snow (ds), (ii) metamorphosed snow (mII), (iii) depth hoar (dh), and iv) wet snow (ws), which have been incorporated using X-ray tomography [14]. They represent a broad range of snow types naturally occurring. Besides, based on the exact morphology, four corresponding artificial snow samples were generated with the same porosity and specific area surface, composed of identical overlapping semi-transparent spheres (ds_eq, mII_eq, dh_eq, and ws_eq). The porosity ranges between 0.85 and 0.38 for the ds and ws snow types, respectively, and the characteristic mean ice particle diameter ranges between 50 and $660 \mu \mathrm{m}$ for the ds and ws snow types, respectively [14]. These morphological characteristics (summarized in Table 1) have been previously calculated based on two-point correlation functions (porosity and specific surface), mathematical opening operations (size distributions), and porosity calculations of increasing subvolumes (representative volume) [13]. 
a)

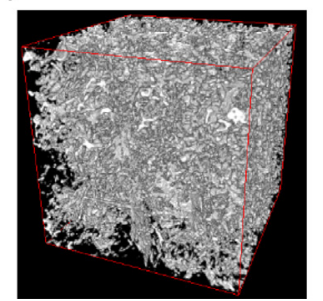

b)

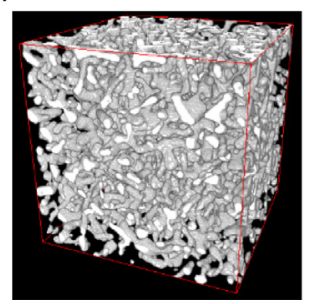

c)

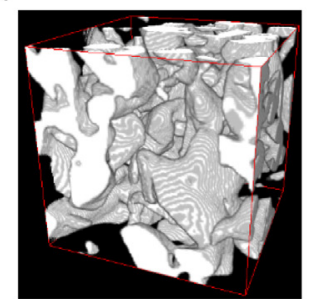

d)

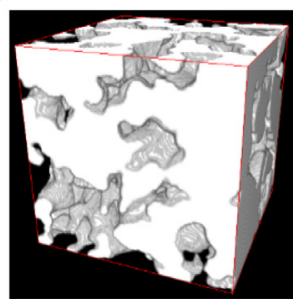

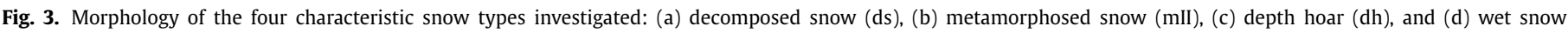
(ws). All samples have an edge length of $4 \mathrm{~mm}$.

a)

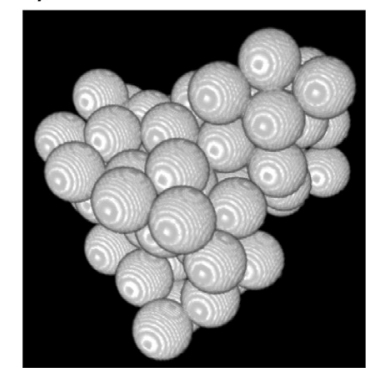

b)

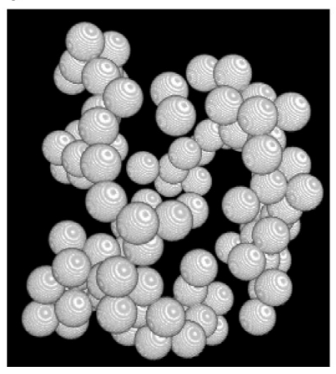

c)

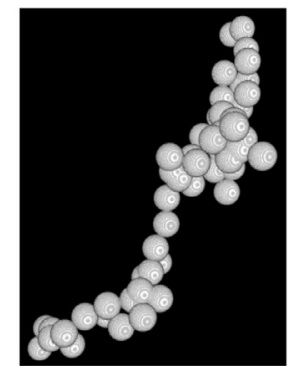

d)

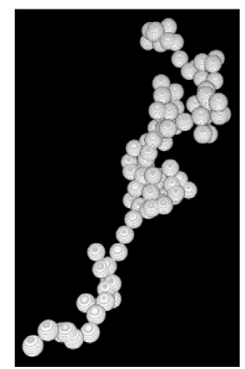

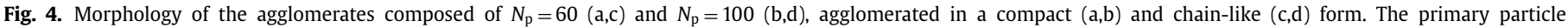
diameter of the agglomerates is $d_{\mathrm{p}}=20 \mathrm{~nm}$.

The soot agglomerates are approximated by a collection of monodisperse, spherical particles with a primary particle diameter size, $d_{\mathrm{p}}$, and composed of a certain number of particles, $N_{\mathrm{p}}$. We chose four distinct soot agglomerates (Fig. 4) with: (i) $d_{\mathrm{p}}=20 \mathrm{~nm}$, $N_{\mathrm{p}}=60$, (ii) $d_{\mathrm{p}}=20 \mathrm{~nm}, N_{\mathrm{p}}=100$, (iii) $d_{\mathrm{p}}=40 \mathrm{~nm}, N_{\mathrm{p}}=60$, and (iv) $d_{\mathrm{p}}=40 \mathrm{~nm}, N_{\mathrm{p}}=100$. The corresponding effective radius of the agglomerates is between 39 and $93 \mathrm{~nm}$.

Soot primary particle diameter sizes ranging from $10-30 \mathrm{~nm}$ have been reported for soot generated from diesel combustion systems [37]. Xu et al. [38] measured primary particle sizes in axisymmetric laminar co-flowing jet diffusion flames. The primary particle diameter varied between 10 and $60 \mathrm{~nm}$, dependent on the collection height above the flame, the flame size and characteristic residence time $[39,40]$. In terms of the soot size, depending on various formation processes, $N_{\mathrm{p}}$ covers a large range. Soot particles which were collected from the atmosphere have aerodynamic diameters of 50 to $300 \mathrm{~nm}$ reported by Adachi et al. [41], and $N_{\mathrm{p}}$ ranging from few tens to several thousands.

The aggregates were created by randomly accumulating spherical primary particles, ensuring that each particle has at least one connection with another particle. The resulting agglomerates exhibited a variety of shapes. For each type, two extreme agglomerate shapes were considered: a compact structure and a chainlike structure to span the typically observed agglomerate structures [40]. The fractal dimension ranged between 2.35-2.17 and 2.03-2.08 for the compact and the chain-like agglomerates, respectively, and the anisotropy ranged between $0.186-0.202$ and 0.310-0.302 for compact and chain-like agglomerates, respectively (Table 2). The anisotropy was quantified based on a mean intercept length calculation [13], where anisotropies close to 0 represent isotropic media and anisotropies close to 1 strongly anisotropic media. The experimentally determined fractal dimension of soot particles spans a range between 2.1 (for flame-generated soot particles) and 2.3 (for diesel exhaust particle matter) [42]. The variation of the fractal dimension in a flame was experimentally and numerically investigated by Stasio et al. [43] ranged between 2.7 and 1.2 , dependent on the residence time in the flame. a)

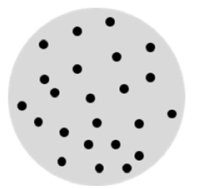

b)

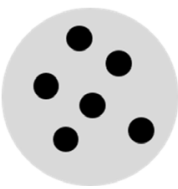

c)

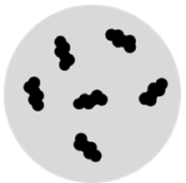

Fig. 5. Modeling approach for agglomerated soot in ice particles of snow: (a) homogeneously distributed primary soot particles, (b) homogeneously distributed agglomerated soot particles modeled by an equivalent larger sphere, and (c) homogeneously distributed agglomerates.

Additionally, we ( $i$ ) approximated the soot agglomerates by an equivalent sphere with the same volume as the agglomerates:

$a_{\mathrm{eff}}=\frac{1}{2} \sqrt[3]{N_{\mathrm{p}}} d_{\mathrm{p}}$

and (ii) by non-agglomerated homogeneously distributed primary soot particles (Fig. 5). As presented in Eq. (8), the effective radius $a_{\text {eff }}$ for the two agglomerates (case c in Fig. 5) is equal to the radius of the equivalent sphere (case b in Fig. 5), while for homogenously distributed primary soot particles (case a in Fig. 5), $a_{\text {eff }}=d_{\mathrm{p}} / 2$. These are also the $a_{\text {eff }}$ used in Eq. (1) for the calculation of the small-scale properties.

The physical properties of soot differ significantly dependent on the chemical composition and formation process. The implemented spectral dependence of the refractive indexes for soot and ice are plotted in Fig. 6. We used two sets of complex refractive indexes, $m$, of soot: $(i)$ the GEISA database [44], originating from measurements Shettle and Fenn [45], and (ii) measurements reported by Chang and Charalampopoulos [46]. At $550 \mathrm{~nm}, m_{1}=1.75+0.44 \mathrm{i}$ and $m_{2}=1.74+0.59$ i. If not indicated otherwise, the data from GEISA and Shettle and Fenn was used [45]. The refractive index of pure ice as reported by Wiscome and Warren was used [3].

\subsection{Small-scale radiative properties}

The calculation of the radiative properties of the soot agglomerates was performed at 55 distinct wavelengths between 
Table 2

Morphological characteristics of soot agglomerates. The type identifier has as the first subscript small ( $20 \mathrm{~nm})$ or large $(40 \mathrm{~nm})$ primary particle diameter, small (60) or large (100) number of particle per agglomerate, and a compact (d) or chain-like (c) agglomerate structure.

\begin{tabular}{|c|c|c|c|c|c|c|}
\hline Type & Primary particle diam. (nm) & Particle number & Porosity & Specific surface $\left(\mu \mathrm{m}^{-1}\right)$ & Effective radius (nm) & Fractional dimension \\
\hline a_ssd & 20 & \multirow{2}{*}{60} & \multirow{2}{*}{0.21} & 50.3 & \multirow{2}{*}{39} & \multirow{2}{*}{2.35} \\
\hline a_lsd & 40 & & & 25.1 & & \\
\hline a_sld & 20 & \multirow{2}{*}{100} & \multirow{2}{*}{0.08} & 21.8 & \multirow{2}{*}{46} & \multirow{2}{*}{2.17} \\
\hline a_lld & 40 & & & 10.9 & & \\
\hline a_ssc & 20 & \multirow{2}{*}{60} & \multirow{2}{*}{0.03} & 8.1 & \multirow{2}{*}{78} & \multirow{2}{*}{2.03} \\
\hline a_lsc & 40 & & & 4.0 & & \\
\hline a_slc & 20 & 100 & 0.03 & 7.8 & 93 & 2.08 \\
\hline
\end{tabular}

a)

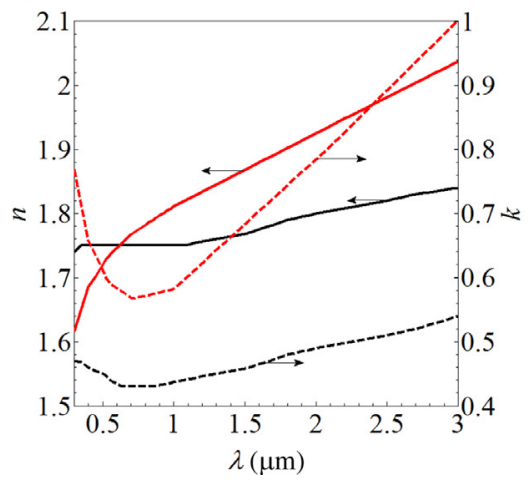

b)

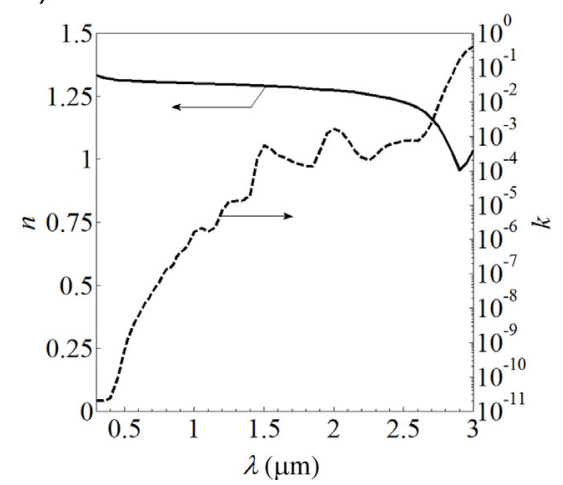

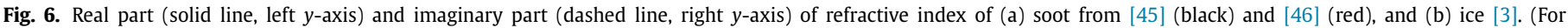
interpretation of the references to colour in this figure legend, the reader is referred to the web version of this article.)

$0.3 \mu \mathrm{m}$ and $2.5 \mu \mathrm{m}(\Delta \lambda=0.05 \mu \mathrm{m}$ for $0.3 \mu \mathrm{m}<\lambda \leq 0.5 \mu \mathrm{m}$ and $1 \mu \mathrm{m}<\lambda \leq 2.5 \mu \mathrm{m}$, and $\Delta \lambda=0.025 \mu \mathrm{m}$ for $0.5 \mu \mathrm{m}<\lambda \leq 1 \mu \mathrm{m})$.

Fig. 7 shows the predicted spectral extinction, absorption efficiency factors, ratios between scattering extinction efficiency factors, $Q_{s} / Q_{e}$, and the asymmetry factor, $g$, all as a function of the wavelength, $\lambda$, calculated by DDSCAT $[23,24,27]$ and BHMIE [22] for the four soot agglomerate models (compact agglomerate, chain-like agglomerate, equivalent sphere, and primary particles, Fig. 5). We show the results for distinct sets of soot agglomerates with different values of $m, d_{\mathrm{p}}$ and $N_{\mathrm{p}}$. The set of soot with $m_{1}$, $d_{\mathrm{p}}=20 \mathrm{~nm}$ and $N_{\mathrm{p}}=60$ was chosen as reference case in this section. The calculation grid spacing, $s$, used was $7 \mathrm{~nm}$, satisfying the validity criteria for DDA, $|m| \omega s \leq 1$ [47,48]. In addition, the results were the averaged values over 64 different calculations obtained by randomly varying target orientations $[29,49]$.

We used the original Lorenz-Mie theory (applicable for nonabsorbing ambient medium) instead of the generalized LorenzMie theory (applicable for absorbing ambient medium) for the full wavelength range. For small wavelengths $(<2.5 \mu \mathrm{m})$ the imaginary part of the refractive index of ice is significantly smaller than the absorption of soot, resulting in differences between original and generalized Lorenz-Mie results of less than $0.01 \%$. When ice phase becomes highly absorbing $(\lambda>2.5 \mu \mathrm{m})$, the generalized Lorenz-Mie theory should be considered. However, in this case, the influence of the soot particle is negligible and the absorption in the ice is dominating (see Fig. 9a).

The aggregate structure and size, and monomer size affected the magnitude of $Q_{e}$ but not the trend with respect to $\lambda$. The aggregate composed of particles with doubled primary particle diameter $\left(d_{\mathrm{p}}=40 \mathrm{~nm}, N_{\mathrm{p}}=60\right)$ yielded around 1.7 to 2 times larger $Q_{\mathrm{e}}$ at $\lambda=0.3 \mu \mathrm{m}$ than the soot particles in reference case $\left(d_{\mathrm{p}}=20 \mathrm{~nm}\right.$, $N_{\mathrm{p}}=60$ ). The effect of increasing number of particles per aggregate $\left(d_{\mathrm{p}}=20 \mathrm{~nm}, N_{\mathrm{p}}=100\right.$ instead of $\left.N_{\mathrm{p}}=60\right)$ was less significant, only about $20 \%$ increase in $Q_{e}$ was observed. The different sensitivity in the extinction properties is associated with the different increase in the effective size, or effective radius $a_{\text {eff }}$, for soot agglomerates in the reference case (for $d_{\mathrm{p}}=20 \mathrm{~nm}, N_{\mathrm{p}}=60, a_{\text {eff }}=39 \mathrm{~nm}$ ), which is doubled for soot of $d_{\mathrm{p}}=40 \mathrm{~nm}$ and $N_{\mathrm{p}}=60$ (i.e. $a_{\mathrm{eff}}=78 \mathrm{~nm}$ ), while $a_{\text {eff }}$ is increased by only $18 \%$ for soot of $d_{\mathrm{p}}=20 \mathrm{~nm}$ and $N_{\mathrm{p}}=100$ (to $a_{\mathrm{eff}}=46 \mathrm{~nm}$ ). This implies a possible monotonic relation between $Q_{\mathrm{e}}$ and $a_{\mathrm{eff}}$, namely that $Q_{\mathrm{e}}=c \cdot a_{\mathrm{eff}}$ where $c$ is a morphology-dependent coefficient which accounts for the effect of the exact structure on the optical properties. Variation of the soot bulk properties, i.e. $m$, shows an obvious dependency of the radiative properties on the exact value of $m$ and also affects the wavelength-dependent evolution of the properties. Similarly, it has been reported in the literature that the effect of chemical composition of primary particle may have strong impact on the mass absorption cross section for the complete soot ensemble [50].

$Q_{a}$ was exponentially decreasing with increasing wavelength and shows a similar proportional relationship between $Q_{a}$ and $a_{\text {eff }}$. This size dependent behavior has also been described by Kahnert's mass absorption cross section [51]. The morphology-dependent behavior of $Q_{a}$ was generally less important compared to $Q_{e}$ over the investigated wavelength range for most soot cases. This finding is consistent with the statement of Radney et al., who reported experimental data with insignificant differences in the absorption cross sections between different morphologies in soot particles at wavelength of $405 \mathrm{~nm}$ [52]. Here, an evident difference was observed for the soot set of $d_{\mathrm{p}}=40 \mathrm{~nm}$ and $N_{\mathrm{p}}=60$ for wavelengths below $0.5 \mu \mathrm{m}$ : compact aggregated soot yielded $14 \%$ higher $Q_{a}$ than volume-equivalent spherical soot, and chain-like aggregated soot absorbed $11 \%$ more than compact aggregates. This finding is in agreement with what has been observed by Kahnert and Devasthale [53] and Scarnato et al. [49].

The scattering interaction was decreasing with increasing wavelength, where, in turn, absorption dominated. We observed that the scattering efficiency was significantly overestimated (compared to agglomerates) when the soot was modeled as an equivalent sphere, independent of $d_{\mathrm{p}}$ or $N_{\mathrm{p}}$. Generally, soot agglomerates with densely packed bodies tend to scatter more, owing to the enhanced scattering interaction between monomers. This difference was fur- 
a)

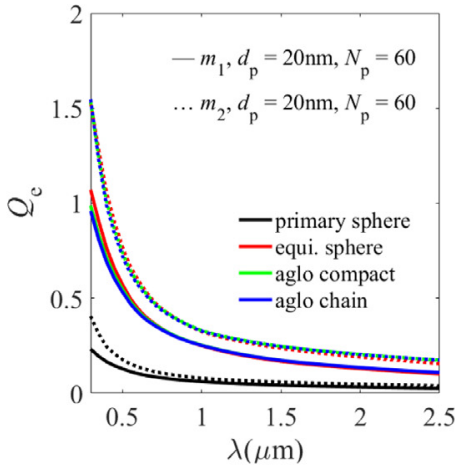

b)

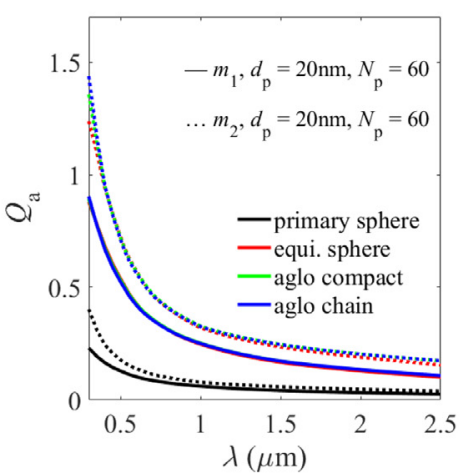

c)

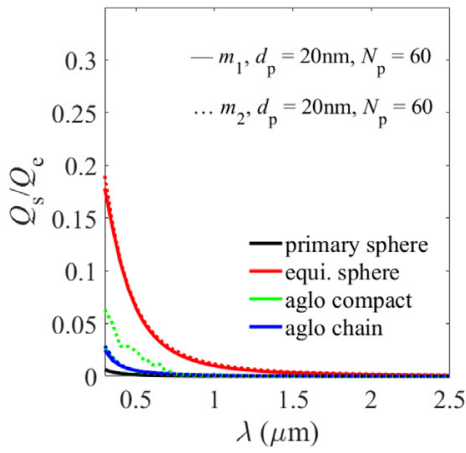

d) I.

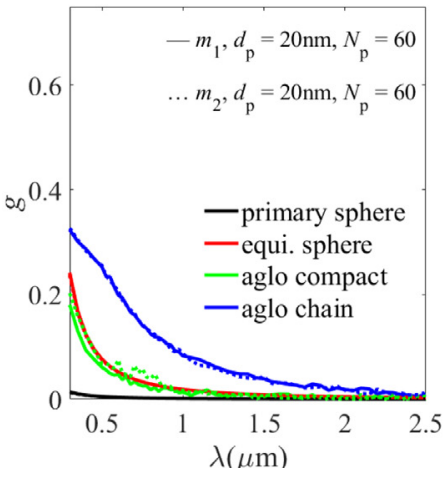

II.

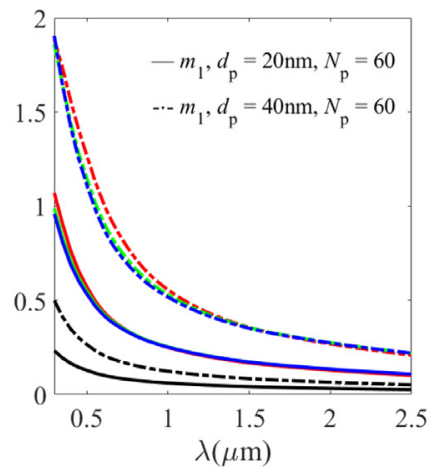

II.

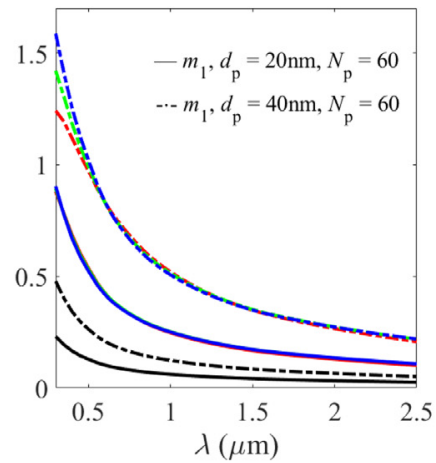

II.

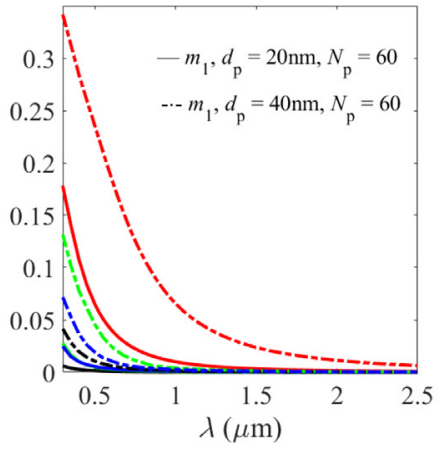

II.

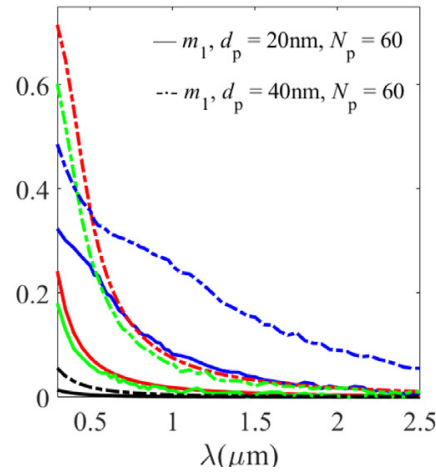

III.

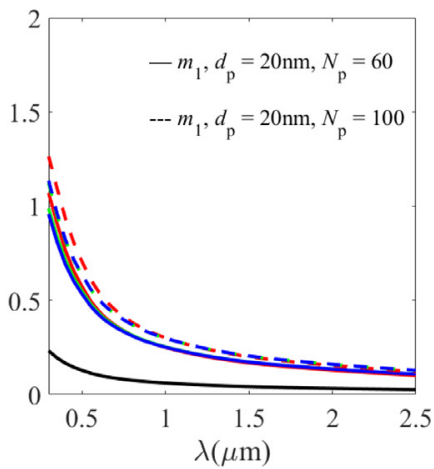

III.

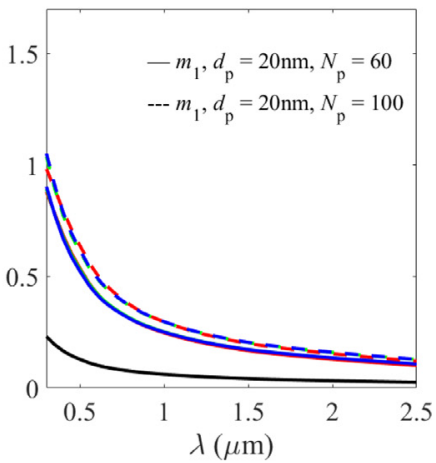

III.

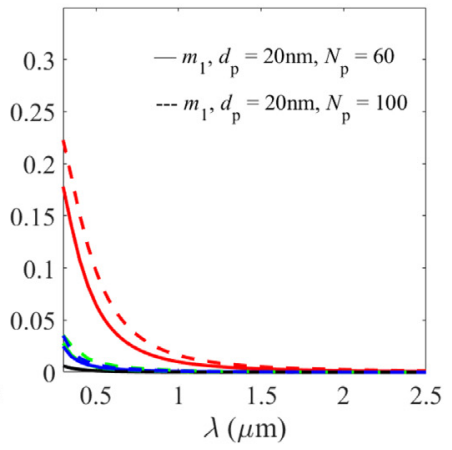

III.

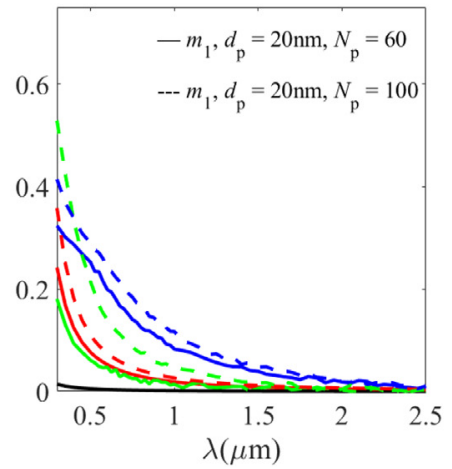

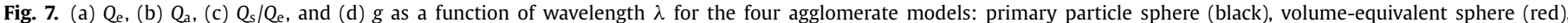

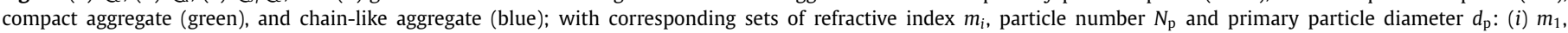

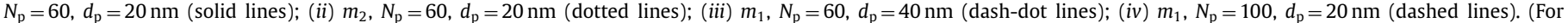
interpretation of the references to colour in this figure legend, the reader is referred to the web version of this article.) 
a)

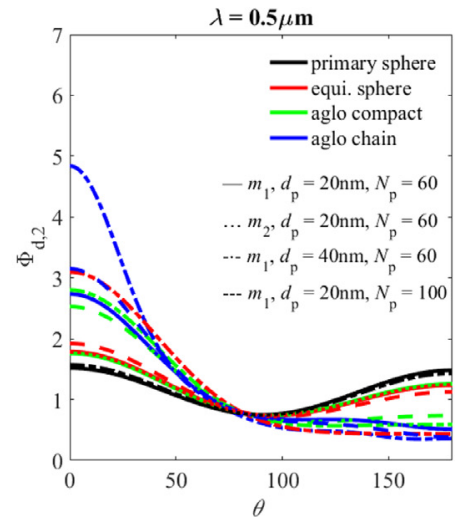

b)

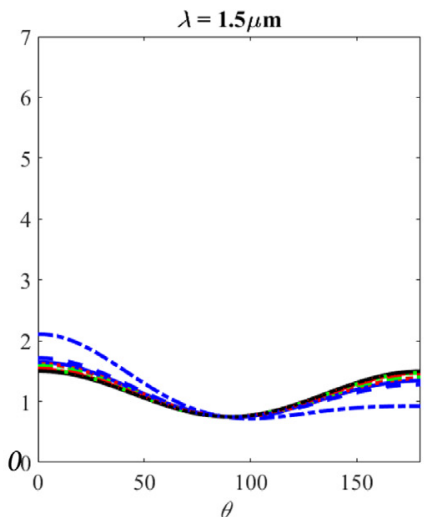

c)

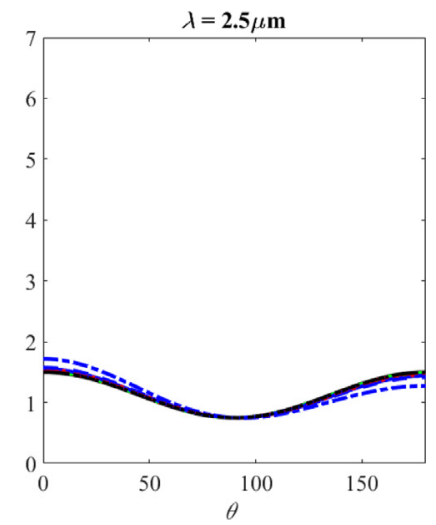

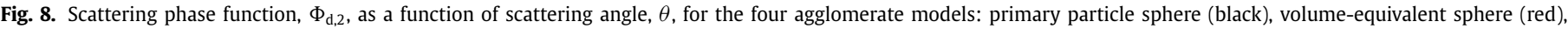

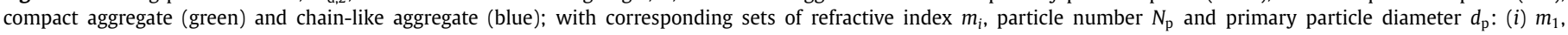

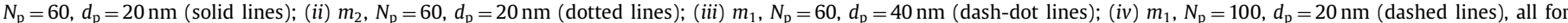

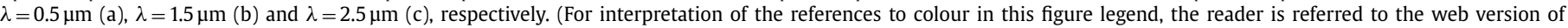
this article.)

ther enlarged for the soot aggregates with larger primary diameter: the ratio of $Q_{s} / Q_{e}$ increased by $85 \%$ at $\lambda=0.3 \mu \mathrm{m}$ for compact aggregates compared to chain-like agglomerates (for $d_{\mathrm{p}}=40 \mathrm{~nm}$ and $N_{\mathrm{p}}=60$ ). In addition, an increase of $46 \%$ in $Q_{s} / Q_{e}$ at $\lambda=0.3 \mu \mathrm{m}$ was observed for soot aggregates composed of more particles (increasing $N_{\mathrm{p}}$ from 60 to 100 , both for $d_{\mathrm{p}}=20 \mathrm{~nm}$ ). Liu et al. [54] have reported the same tendency in the simulated scattering cross section and $Q_{s} / Q_{e}$ at a certain wavelength by using the T-matrix method for a series of fractal aggregates samples.

For all modeled configurations and morphologies, $g$ was positive in the considered spectrum, indicating that more light is scattered in the forward direction. Generally, $g$ approached zero with increasing wavelength. For the soot made of $d_{\mathrm{p}}=20 \mathrm{~nm}, g$ was close to zero $(g=0.01$ at $\lambda=0.3 \mu \mathrm{m}$ and $g=0.002$ at $\lambda=2.5 \mu \mathrm{m}$ ), which implies the scattering is almost isotropic or the scattering is symmetric about a scattering angle of $90^{\circ}$ [22]. For the smallest sized soot at reference condition $\left(d_{\mathrm{p}}=20 \mathrm{~nm}\right.$ and $\left.N_{\mathrm{p}}=60\right), \mathrm{g}$ of the soot modeled as equivalent sphere was similar to $g$ of the soot modeled as compact agglomerate, and both were decreasing exponentially with the increasing $\lambda . g$ of the soot modeled as chain-like agglomerate had a much larger $g$ (1.8 times larger than compact agglomerates at $\lambda=0.3 \mu \mathrm{m}$ ) and followed a decreasing trend, about $40 \%$ to $60 \%$ "slower" than the other two agglomerate models. Similar trends were observed for increasing primary particle sizes or agglomerates with increased particle number. Generally, the scattering behavior of soot agglomerates significantly depended on its exact morphology, while the refractive index had no significant impact on it.

The detailed scattering behavior of the agglomerated soot, $\Phi_{\mathrm{d}, 2}$, as a function of the scattering angle, $\theta$, at wavelengths $\lambda=0.5,1.5$, and $2.5 \mu \mathrm{m}$ are presented in Fig. 8. We showed the four soot agglomerate models with four sets of combinations of refractive indexes, primary diameter and particle number. For primary spherical soot of small size, at the presented wavelengths, the direction of least scattering appeared at $90^{\circ}$, while the scattering into forward and backward directions was nearly symmetric. When increasing the soot size, either by extending the primary diameter or by adding more particles, the scattering became non symmetric about $90^{\circ}$ Instead the forward scattering in the range between 0 to $90^{\circ}$ was enhanced while the backward scattering in the range between 90 to $180^{\circ}$ was decreased. However, while generally the forward scattering was more pronounced than backward scattering, the latter was not insignificant, the positive $g$ results from a symmetric scattering about $90^{\circ}$ rather than a dominance of forward scattering.

Scarnato et al. [49] also has predicted that increasing particle size leads to a rise in forward scattering. The largest morphology-related differences in $\Phi_{\mathrm{d}, 2}$ were observed at a short $\lambda$ of $0.5 \mu \mathrm{m}$. The chain-like agglomerated soot scatters around $57 \%$ more light than compact agglomerated soot at an angle of $0^{\circ}$ for $d_{\mathrm{p}}=20 \mathrm{~nm}$ and $N_{\mathrm{p}}=60,50 \%$ for $d_{\mathrm{p}}=40 \mathrm{~nm}$ and $N_{\mathrm{p}}=60$, and $22 \%$ for $d_{\mathrm{p}}=20 \mathrm{~nm}$ and $N_{\mathrm{p}}=100$. With increasing size, the difference in $\Phi_{\mathrm{d}, 2}$ due to morphology decreased. Additionally, this difference is also decreasing with the increasing $\lambda$. Therefore, we concluded that lacy, chain-like structures tends to have stronger forward scattering than compact structures. The scattering phase function showed no sensitivity to the refractive index.

After adding soot impurities into the bulk ice phase, the new bulk properties of the ice-soot mixture will change according to Eqs. (1)-(3). To evaluate the impact of soot impurities on the mixed ice-soot phase, we present one example case, assuming the added soot agglomerate is composed of particles of $d_{\mathrm{p}}=20 \mathrm{~nm}$ and $N_{\mathrm{p}}=60$, with a volume fraction of $f_{v}=10^{-6}$. Fig. 9 shows the extinction coefficient, $\beta_{\mathrm{d}, 2}$, and the scattering albedo, $\sigma_{\mathrm{s}, \mathrm{d}, 2} / \beta_{\mathrm{d}, 2}$, of the ice-soot mixture. In the long wavelength range, the extinction of the ice-soot mixture is completely dominated by the ice absorption, addition of soot has no effect on the mixed icesoot phase. However, soot impurities significantly affect $\beta_{\mathrm{d}, 2}$ and $\sigma_{\mathrm{s}, \mathrm{d}, 2} / \beta_{\mathrm{d}, 2}$ in the short wavelength range $(\lambda<1.4 \mu \mathrm{m})$. The soot impurities increase $\beta_{\mathrm{d}, 2}$ and introduce scattering to the ice-soot phase. $\beta_{\mathrm{d}, 2}$ increased from 0.0008 to $212 \mathrm{~m}^{-1}$ at $\lambda=0.3 \mu \mathrm{m}$, representing the largest possible increase. Among different soot morphologies, equivalent spherical soot is the most scattering one. An obvious difference was found in $\sigma_{\mathrm{s}, \mathrm{d}, 2} / \beta_{\mathrm{d}, 2}$ between compact and chain-like agglomerated soot (Fig. 9): the compact soot scatters about $70 \%$ more than chain-like soot agglomerate over the short range of wavelengths (up to $\lambda=0.6 \mu \mathrm{m}$ ) investigated. This finding is in accordance with the previously presented results of the efficiency ratio, and is due to the enhanced interaction between the particles arranged closer together for the compact agglomerated soot than the chain-like agglomerated soot [54,55].

\subsection{Medium-scale radiative properties}

As the two-phase medium effective properties, $\sigma_{\mathrm{s}, \text { int }, i i}, \sigma_{\mathrm{s}, \mathrm{int}, i j}$, $\Phi_{\text {int }, i i}, \Phi_{\text {int }, i j}$, by definition, are solely dependent on the snow mor- 
a)

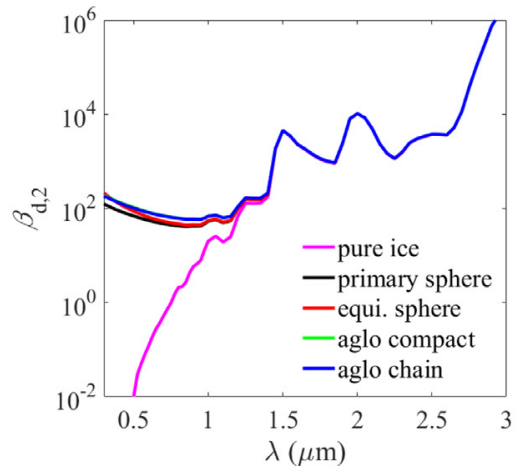

b)

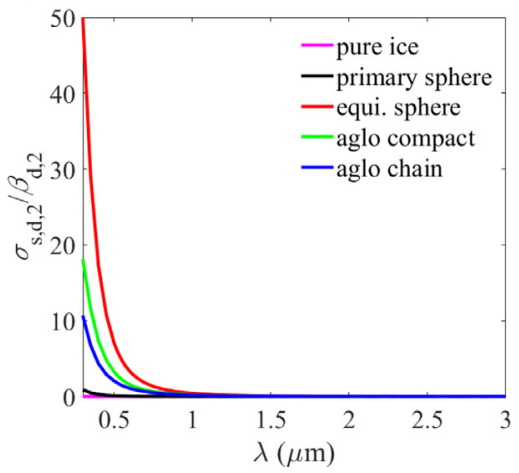

Fig. 9. (a) $\beta_{\mathrm{d}, 2}$ and (b) $\sigma_{\mathrm{s}, \mathrm{d}, 2} / \beta_{\mathrm{d}, 2}$ of pure ice and ice with soot agglomerates composed of $N_{\mathrm{p}}=60$ and $d_{\mathrm{p}}=20 \mathrm{~nm}$, for $f_{v}=10^{-6}$ in a wavelength range of $0.3 \leq \lambda \leq 3 \mu \mathrm{m}$.

a)

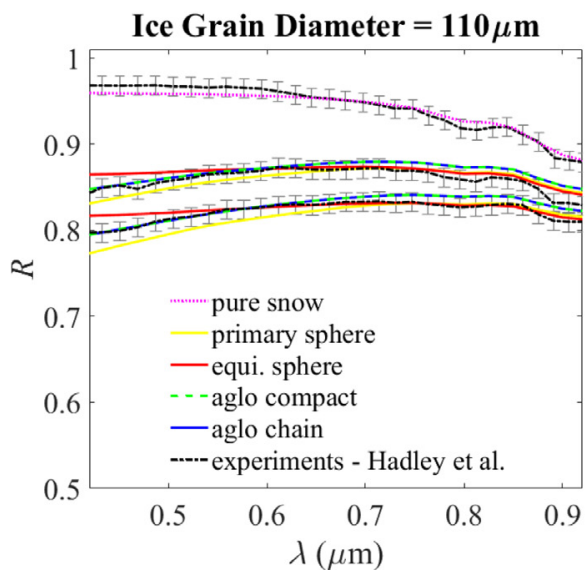

b)

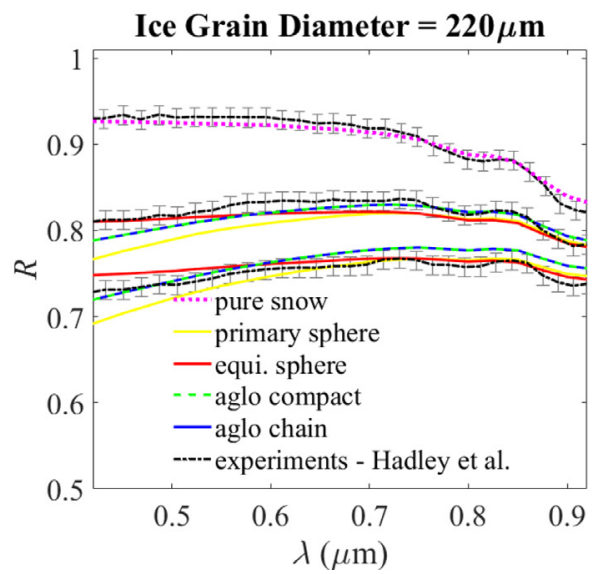

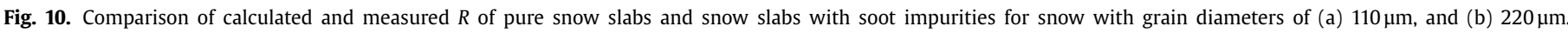

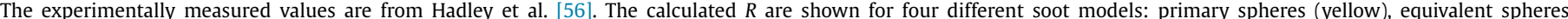

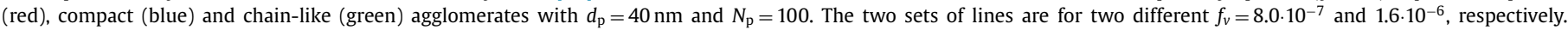

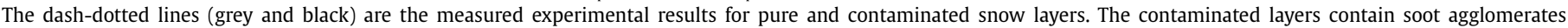

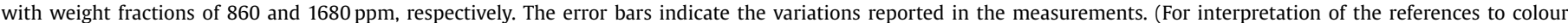
in this figure legend, the reader is referred to the web version of this article.)

phology, they are equivalent to what has been reported previously for these snow types [14]. $\sigma_{\mathrm{s}, \text { int,ij }}$ and $\sigma_{\mathrm{s}, \text { int,ii }}$ are closely linked to the interface reflection behavior and, consequently, $\sigma_{\mathrm{s}, \text { int,ij }}$ is mirroring the behavior of $\sigma_{\mathrm{s}, \text { int }, i i} \cdot \sigma_{\mathrm{s}, \text { int,21 }}$ is additionally influenced by the presence of total reflection phenomena in the ice particle. $\beta_{1}$ is independent of $\lambda$ because it is a function of the interface geometry only as the gas phase is transparent. $\sigma_{\mathrm{s}, 22}, \beta_{2}$ and $\Phi_{22}$ include also the bulk radiative behavior of the mixed ice-soot phase (phase 2 ) and therefore are changing when soot impurities are added, following the same trends of the corresponding soot properties at the small-scale. $\beta_{2}$ follows qualitatively the $\lambda$-dependence of $\beta_{\mathrm{d}, 2}$. For $\beta_{2}, \lambda<1.4 \mu \mathrm{m}$, the role of soot impurities is dominant; $\lambda>1.4 \mu \mathrm{m}$, the influence of ice absorption becomes relevant to $\sigma_{\mathrm{s}, 21}$. Therefore, $\beta_{2}$ calculated by Monte-Carlo simulation follows qualitatively the $\lambda$-dependence of $\beta_{\mathrm{d}, 2}$.

\subsection{Macroscopic optical properties}

The macroscopic optical properties, $R, \operatorname{Tr}$ and $A$, of a snow slab of $4 \mathrm{~cm}$ thickness were calculated by using the previously determined small- and medium-scale optical properties, which account for the complex geometry of the snow layer and the agglomerated soot.

As listed in Table 1, four exact characteristic snow types and four corresponding simplified morphological snow types are con- sidered. We explored the change of the macroscopic optical properties of snow slabs when soot impurities are present.

\subsubsection{Experimental validation}

We validated our multi-scale model by comparing the simulated results with the experimental results reported by Hadley et al. [56] under the same condition, specifically their measured reflectance, $R$, for $0.4 \mu \mathrm{m}<\lambda<1 \mu \mathrm{m}$ (Fig. 10).

The pure snow samples they measured were densely packed snow layers of $2 \mathrm{~cm}$ thickness with a density $0.55 \mathrm{~kg} \mathrm{~m}^{-3}$ made by freezing droplets of water. Consequently, their snow type was composed of spherical ice particles. The optical effective diameters of the spherical ice grains were 110 and $220 \mu \mathrm{m}$, respectively. In order to have the same pure snow layer, we generated pure snow utilizing monodisperse non-overlapping spherical (MNOS snow) ice grains with the identical grain diameter, thickness and density as in their experimental setup. The comparison results are plotted separately for snow samples with two sizes (Fig. 10). A good agreement between our calculated $R$ and their experimental measurement was obtained. The experimental data used here was extracted from the origin data without their adjustment factor [56]. Hadley et al. used this factor to fit their experimental results with the results of the Snow, Ice and Aerosol Radiation (SNICAR) model [57]. This factor, correcting for their different geometry $(2 \mathrm{~cm}-$ depth in the experiment and infinitely deep slab for the SNICAR 
model), is not needed in our case as we model the same geometry that was experimentally investigated.

For the validation of the model for contaminated snow layer, we needed to adjust our soot properties in order to fit them with the experimentally used soot. The mass absorption coefficient (MAC) for the soot impurities at a certain wavelength, $\mathrm{MAC}=3 Q_{\mathrm{abs}} /\left(4 \rho_{\text {soot }} a_{\text {eff }}\right)$, is introduced. Hadley et al. experimentally measured a MAC of $15 \pm 1 \mathrm{~m}^{2} \mathrm{~g}^{-1}$ at $\lambda=532 \mathrm{~nm}$, and reported a MAC range of $7.5-15 \mathrm{~m}^{2} \mathrm{~g}^{-1}$ for similar soot.

We chose two of their reported cases with concentrations (g.m ${ }^{-1}$ ) of impurities inside the snow of 860 and $1680 \mathrm{ppm}$. We used soot agglomerates with $N_{\mathrm{p}}=100, d_{\mathrm{p}}=40 \mathrm{~nm}$, and $m_{2}$ for the comparison. The corresponding MAC values of the chosen soot at $\lambda=550 \mathrm{~nm}$ with an assumed soot density of $1 \mathrm{~g}^{1} \mathrm{~cm}^{-3}$ $[3,4]$ are $11.6 \mathrm{~m}^{2} \mathrm{~g}^{-1}$ for primary spheres, $9.6 \mathrm{~m}^{2} \mathrm{~g}^{-1}$ for volumeequivalent sphere agglomerates, $10.0 \mathrm{~m}^{2} \mathrm{~g}^{-1}$ for compact agglomerates, $10.3 \mathrm{~m}^{2} \mathrm{~g}^{-1}$ for chain-like agglomerates, respectively.

The colored lines in Fig. 10 represent the averaged simulated results (based on three randomly generated MNOS morphologies) for pure snow layers and contaminated snow layers containing the corresponding soot morphology types. The upper set of lines is for snow layers including impurities with $f_{v}=8.6 \cdot 10^{-7}$, and the lower set of lines is with $f_{v}=1.68 \cdot 10^{-6}$, which correspond to the measured mass proportion of $860 \mathrm{ppm}$ and $1680 \mathrm{ppm}$, respectively.

The experimental and computed results followed the same wavelength-dependence. There was a significant overlap between the experiment and model, indicating that our multi-scale model can qualitatively and quantitatively describe the macroscopic radiative properties of contaminated snow layers. In the low wavelength region $(\lambda<0.65 \mu \mathrm{m})$, the agglomerate models utilizing chain-like and compact agglomerates provide a better agreement than the model utilizing volume-equivalent spheres and primary spheres. The snow containing volume-equivalent spherical soot yields $2 \%$ higher $R$ than that with aggregated soot at $\lambda=0.4 \mu \mathrm{m}$. The extra reflection for the equivalent sphere agglomerates resulted from the enhanced scattering, and the lower absorption (see Section 4.3). The snow containing primary spherical soot has $2 \%$ lower $R$ compared to the two aggregated soot models at this wavelength $(\lambda=0.4 \mu \mathrm{m})$. This is due to higher cross section (MAC) yielded by primary spherical soot at short wavelengths. The crossover of $R$ for the various soot models at $\lambda \approx 0.65 \mu \mathrm{m}$ follows from the same observation as for the small scale radiative properties (see Section 4.3). At larger wavelengths, absorption in the ice grain starts to dominate, leading to lower reflectance of the snow layers and less sensitivity of the behavior to the agglomerate model.

The small discrepancy between the calculated $R$ and experimentally measured $R$ is expected due to the simplified soot assumption, namely homogeneous distribution of the soot agglomerates and uniform agglomerate morphology. However, the soot contained inside the snow layer can exhibit a range of morphologies and sizes $\left(N_{\mathrm{p}}, d_{\mathrm{p}}\right)$ [58], and a range of bulk properties, all resulting from variations in the formation process. Additionally, soot has a tendency to move towards the ice grain boundaries in both dry and wet snow, leading to a less homogeneous soot distribution in the ice grain [58].

The sensitivity of the results with respect to the choices in soot concentration, primary particle size, and soot type are shown in Fig. 11. As an example, we chose the snow layer with a small grain size of diameter $110 \mu \mathrm{m}$ at an incident wavelength of $\lambda=0.5 \mu \mathrm{m}$ for $N_{\mathrm{p}}=100$ and refractive index $m_{2}$.

$R$ was not equally sensitive to $f_{\mathrm{v}}, d_{\mathrm{p}}$, and morphology. Generally with an increasing $f_{\mathrm{v}}$, the $R$ of mixed snow layer was decreasing. At a soot content of $f_{\mathrm{v}}=10^{-7}$, doubling $d_{\mathrm{p}}$ resulted in increased $R$ by $1.4 \%$ for agglomerated particles and $3.9 \%$ for spherical equivalent spheres. This increase was enlarged when increasing the volume fraction: for agglomerated particles compared to $R$

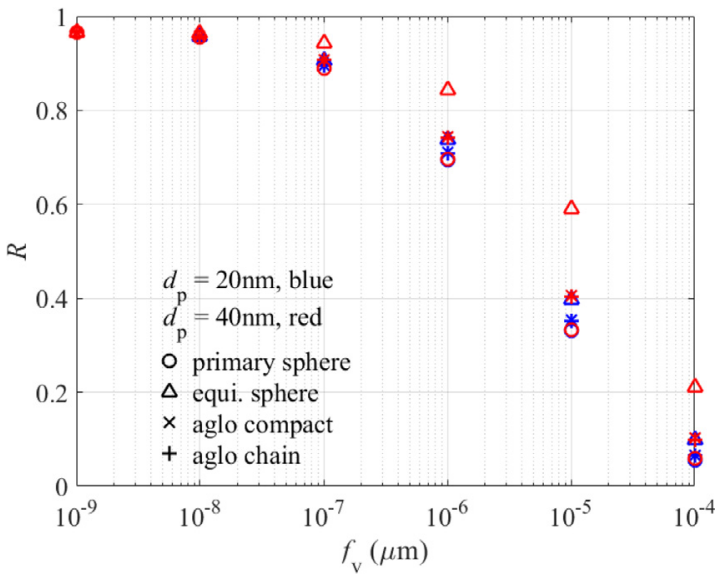

Fig. 11. $R$ as a function of volume fraction, $f_{\mathrm{v}}$, for soot particles of different morphology and with different primary particle diameter.

at $f_{\mathrm{v}}=10^{-7}$, it was 3 times larger for $f_{\mathrm{v}}=10^{-6}, 9$ times larger for $f_{\mathrm{V}}=10^{-5}$, and 23.9 times larger for $f_{\mathrm{v}}=10^{-4}$. Similar trends were observed for equivalent spherical particles: the difference in $R$ with doubled primary diameter compared to $f_{\mathrm{V}}=10^{-7}$ was 3.3 times larger for $f_{\mathrm{V}}=10^{-6}, 8.4$ times larger for $f_{\mathrm{V}}=10^{-5}$, and 13.7 times larger for $f_{\mathrm{V}}=10^{-4}$. The equivalent spherical soot always showed smaller effect on $R$ than aggregated one. While only subtle difference between various agglomerates was found, compact agglomerated soot generally showed higher reflection than chain-like agglomerates.

\subsubsection{Sensitivity to snow morphology}

Fig. 12 shows the evolution of $R$ and $A$ for a $4 \mathrm{~cm}$-thick snow slab made of the four different snow types with identical combination $\left(N_{\mathrm{p}}=60\right.$ and $\left.d_{\mathrm{p}}=20 \mathrm{~nm}\right)$ and volume fraction $\left(f_{\mathrm{v}}=10^{-6}\right)$ of soot impurities.

The different characteristic snow slabs show different magnitudes in $R$ but similar spectral dependencies. $R$ decreases for ds, $\mathrm{mII}$, dh to ws snows, according to their decreasing porosity and increasing ice grain size. The addition of soot impurities lowered $R$ and $T r$, and increased $A$. The macroscopic optical properties follow the evolution of $\beta_{\mathrm{d}, 2}$ with respect to $\lambda$. The most important effect of the soot impurities on the macroscopic optical behavior is observed in the short wavelength range $(0.3 \mu \mathrm{m}<\lambda<1.4 \mu \mathrm{m})$, while they don't affect $R, T r$, and $A$ in the longer wavelength range $(\lambda>1.4 \mu \mathrm{m})$ where the absorption of the ice dominates. Although the amount of added soot impurities is equal for all snow types, they differently affect the macroscopic properties for the different snow types. The reduction of the reflectance $R$ is about $34 \%$ and $44 \%$ for ds and mII snow, respectively, whilst it can reach $68 \%$ and $79 \%$ for $\mathrm{dh}$ and ws, respectively, at $\lambda$ of $0.3 \mu \mathrm{m}$. The same behavior is found for $A$. The enhancement of $A$ in $\mathrm{dh}$ and ws was more important than in ds and mII. At $\lambda=0.3 \mu \mathrm{m}, A$ increases from 0 to $0.38,0.47,0.7$, and 0.81 for $\mathrm{ds}, \mathrm{mII}$, $\mathrm{dh}$, and ws, respectively. The radiative properties of snow with larger ice particle sizes were more sensitive to the addition of soot impurities. These results are in accordance with the predictions by Warren and Wiscombe [4], reporting that the reduction of albedo in coarse-grained snow is greater than in fine-grained snow for a given concentration of soot.

\subsubsection{Sensitivity to soot morphology}

We focus on discussing the macroscopic optical properties within the spectrum from $0.3 \mu \mathrm{m}$ to $1.4 \mu \mathrm{m}$ where the soot impurities most importantly influence the radiative behavior of the snow layers. The calculated spectral $R$ of a snow slab containing 
a)

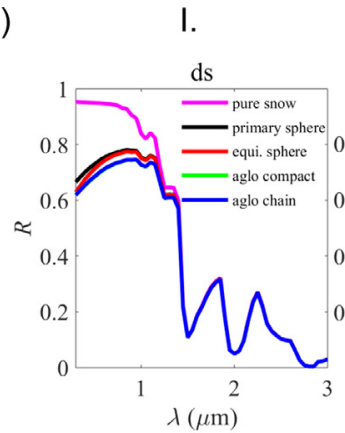

b)

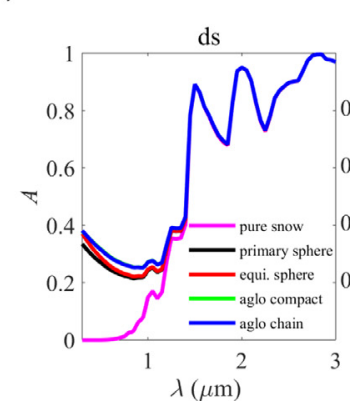

II.

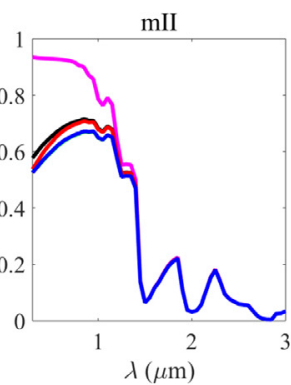

II.
III.

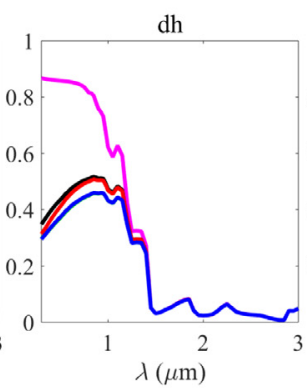

III.
IV.

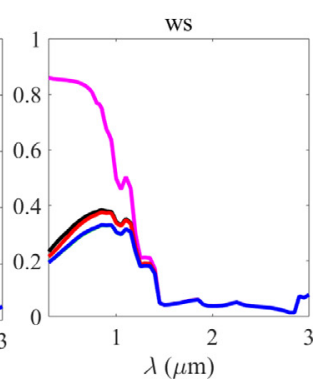

IV.
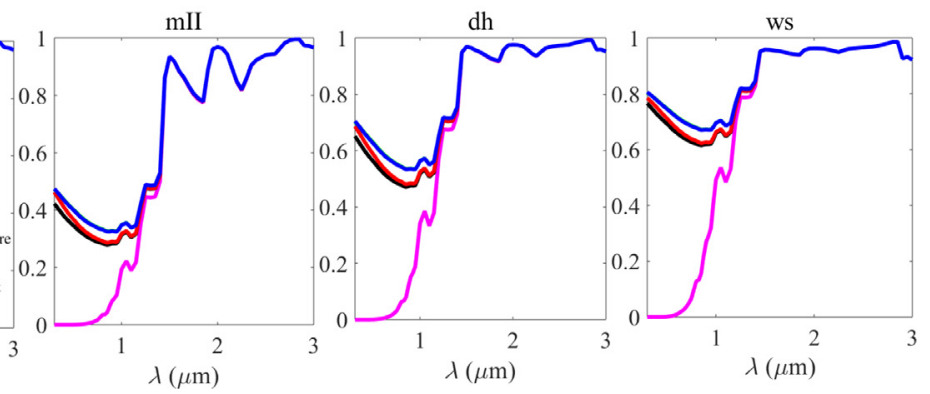

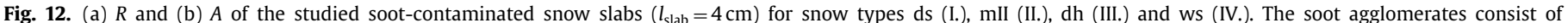
$N_{\mathrm{p}}=60$ and $d_{\mathrm{p}}=20 \mathrm{~nm}$ with $f_{\mathrm{v}}=10^{-6}$. The radiative properties of clean snow are shown as reference.

soot agglomerates made of different combinations of $m, d_{\mathrm{p}}, N_{\mathrm{p}}$ and $f_{\mathrm{v}}$ for snow type ds (high porosity and fine grained snow) and ws (low porosity and coarse grained snow) is shown in Fig. 13. Significant variations in $R$ can be observed for the different soot impurities and morphologies, with magnitudes dependent on the snow morphology. Generally, the largest $R$ is observed for soot particles modeled as homogenously distributed primary spheres, consistent with the calculated small and medium scale properties. Soot modeled as equivalent sphere showed the second largest $R$. Soot agglomerates made by chain and compact agglomerates decreased $R$ the most. For the smallest agglomerates $\left(d_{\mathrm{p}}=20 \mathrm{~nm}, N_{\mathrm{p}}=60\right)$ at $0.3 \mu \mathrm{m}$, equivalent spherical soot and agglomerated soot decreased $R$ by $5 \%$ and $7 \%$ with respect to snow containing primary spherical soot impurities, respectively, for fine-grained ds snow. In comparison, the reduction of $R$ was $8 \%$ and $16 \%$ in the coarse-grained ws snow. By enlarging the primary diameter of the soot or adding more soot particles to increase the agglomerate size, the decrease in $R$ was generally enhanced. However, additional observation was made for the soot with $d_{\mathrm{p}}=40 \mathrm{~nm}$ and $N_{\mathrm{p}}=60$ in the spectrum range near $0.3 \mu \mathrm{m}$ : the effect of equivalent-volume spherical soot on $R$ reduction was suppressed at small $\lambda(<0.5 \mu \mathrm{m})$, and enhanced at large $\lambda$, which resulted from the contribution of higher scattering and lower absorption of equivalent-volume spherical soot at short spectrum (see Section 4.3).

The soot with compact shape yielded $2 \%$ higher $R$ than the one with chain-like shape within ds snow. This is consistent with the tendency we found in small and medium scale radiative properties, compact soot scattered more and absorbed less than chainlike soot. A more pronounced difference of $3 \%$ between the compact and chain-like aggregates on $R$ was observed for ws snow. The coarse-grain snow amplified the difference of $R$ due to the changing morphology of soot impurities.

$R$ was sensitive to the amount of soot $\left(f_{\mathrm{v}}\right)$ and optical characteristics $(m)$. By decreasing the soot content inside the snow from $f_{\mathrm{v}}=10^{-6}$ to $10^{-7}, R$ was reduced by $37 \%$ and $157 \%$ at $\lambda=0.3 \mu \mathrm{m}$ for ds and ws snow, respectively. At the same wavelength when the refractive index of soot impurities was changed from $m_{1}$ to $m_{2}, R$ was shifted in by average $30 \%$ and $15 \%$ for ds and ws snow, respectively.

\section{Summary and conclusion}

We developed a methodology to optically characterize heterogeneous media composed of morphologically-complex components on two distinct scales. We applied the method to sootcontaminated snow layers and characterized their macroscopic optical properties in a spectral range of $0.3-3 \mu \mathrm{m}$. On the small-scale $(x=\pi \cdot d / \lambda<1)$, inspired by SEM images, the soot impurities were modeled as $(i)$ homogeneously distributed primary spheres, (ii) agglomerates approximated as spheres with agglomerate-equivalent diameter, (iii) compact agglomerates made of a random accumulation of primary spheres, and (iv) chain-like agglomerate made of an accumulation of random primary spheres. The agglomerates were made of a different combinations of primary diameter, $d_{\mathrm{p}}$, and particle number, $N_{\mathrm{p}}$. Lorenz-Mie theory and the discrete dipole approximation (DDA) method were used to predict the optical properties of the agglomerate, namely extinction efficiency, absorption efficiency, scattering efficiency, asymmetry factor, and scattering phase function. On the medium-scale $(x>1)$, the detailed 3D morphology of four characteristic snow types was obtained by X-ray tomography and incorporated into the direct solution of the discrete-scale radiative transfer equations (RTEs) for the determination of the effective extinction and scattering coefficients, and effective scattering phase functions. On the large-scale $(x>>1)$, the two-scale radiative transport properties were incorporated into the coupled multi-phase volume-averaged RTEs, applied to a semi-infinite slab of snow, for the determination of the averaged intensity vector field and heat transfer fluxes, in order to estimate fraction of incident radiation that is reflected, $R$, transmitted, $T$, and absorbed, $A$, in the slab. This model was experimentally validated with reflectance measurement of contaminated snow layers.

Significant differences were found in the calculated macroscopic optical properties of soot-contaminated snow layers, dependent on 
a)

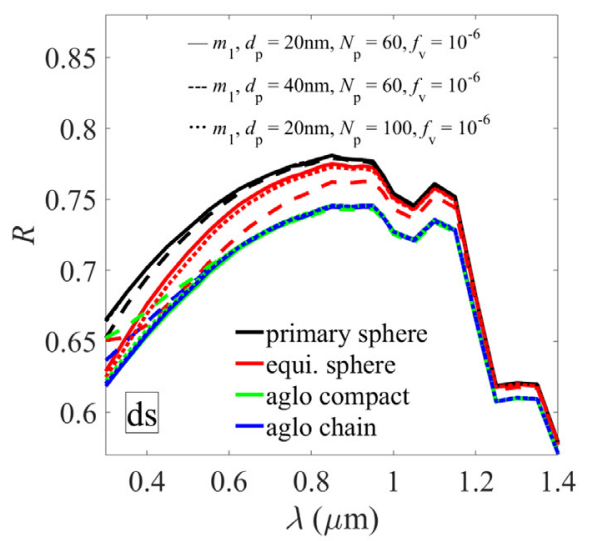

c)

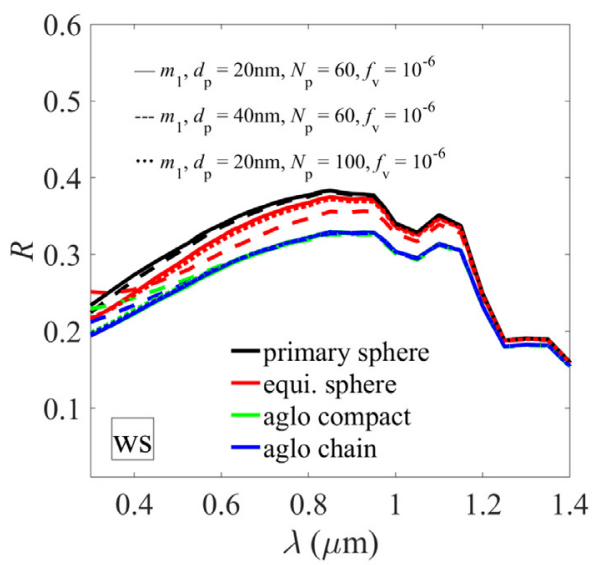

b)

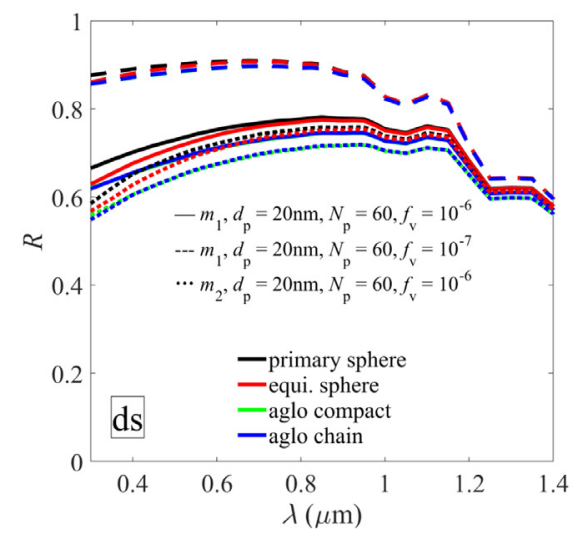

d)

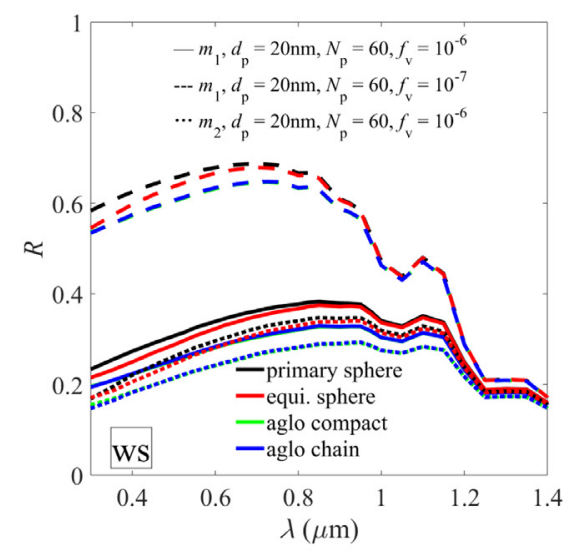

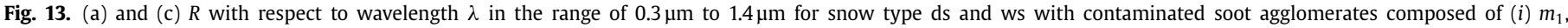

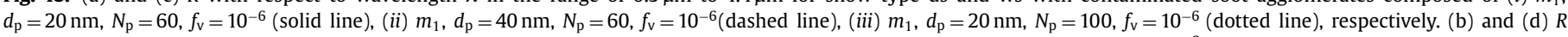

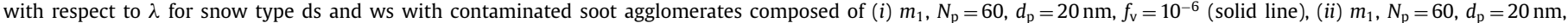
$f_{\mathrm{v}}=10^{-7}$ (dashed line), (iii) $m_{2}, N_{\mathrm{p}}=60, d_{\mathrm{p}}=20 \mathrm{~nm}, f_{\mathrm{v}}=10^{-6}$ (dotted line), respectively.

the approach used to approximate the soot agglomerates. For example, on the small scale, the ratio $Q_{s} / Q_{e}$ of agglomerates approximated as equivalent spheres was 5 times higher than the value calculated for chain and compact-like agglomerates, at $\lambda=0.3 \mu \mathrm{m}$. These results indicated that using Lorenz-Mie theory would not suffice in accurately predicting the radiative characteristics and instead DDA-based methods or the direct solution of the Maxwell's equations, both capable of incorporating the arbitrary shapes and anisotropic physical properties, should be used. Generally, soot in compact agglomerates tended to scatter more but absorb less than chain-like agglomerates. $Q_{s} / Q_{e}$ increased by $85 \%$ for compact agglomerates compared to chain-like agglomerate $(\lambda=0.3 \mu \mathrm{m}$ where the difference was most significant for the spectral range considered). This difference was amplified when increasing the soot size (either by increasing the primary particle size or the number of agglomerated particles). For long wavelengths, the scattering phase function indicated a tendency to be symmetrically distributed around a scattering angle $90^{\circ}$. At short $\lambda$, the scattering was more significant dependent on the morphology and forward scattering was more pronounced. Increasing the primary particle size enhanced forward scattering in all types of soot agglomerates.

On the large scale, a remarkable reduction in $R$ and $T$ and a significant increase in $A$ for all types of snow slabs were observed when soot impurities were added. Those results were in agreement with previously published experimental measurements and calculated predictions of contaminated snow $[4,41,58]$. Moreover, the snow slab with bigger grain size yielded a greater sensitivity to the soot additives and their morphological characteristics (compactness and size). For example, for the presented smallest sized soot $\left(d_{\mathrm{p}}=20 \mathrm{~nm}, N_{\mathrm{p}}=60\right)$, equivalent spherical soot and agglomerated soot yielded $5 \%$ and $7 \%$, respectively, lower $R$ than primary spherical soot (all at $\lambda$ of $0.3 \mu \mathrm{m}$ ) within fine-grained ds snow. In comparison, the extent of reduction was $8 \%$ and $16 \%$, respectively, within coarse-grained ws snow. Moreover, the coarse-grain snow amplified the difference in $R$ due to the changing morphology of soot impurities.

We validated the methodology by comparing the numerical results of the macroscopic optical properties, specifically $R$, with reported experimentally measured $R$. The computational and experimental results overlapped quantitatively and qualitatively with respect $R$ for two different snow types (characterized by two different grain sizes) and two different soot volume fractions, indicating that our presented methodology and its results provide plausible quantification and is predictive for trends. Further improvement could be done, for example, by assuming less uniform soot characteristics and a less homogeneous agglomerate distribution within the ice grain.

The tomography-based, multi-scale methodology proves to be a powerful method to determine the optical characteristics of complex heterogeneous medium, particularly shown here for the radia- 
tive problem in soot contaminated snow slabs. We derive a relation between large-scale radiative properties and small- and mediumscale morphology, which has not been systematically studied before. Our results allow for the de-convolution of different effects on the radiative characteristics, effects resulting from size, morphological and material properties at different scales and for different boundary conditions. In summary, our developed methodology offers a novel approach for the determination of macroscopic optical properties of contaminated snow based on its complex structure on small, medium and large scale, considering a multiphase radiative transfer approach. It allows quantifying the changes in macroscopic radiative properties resulting from changes in morphology of snow and soot on medium and small scale. In general, the approach is applicable to other natural and technical multi-scale media and, therefore, allowed for the investigation of new sets of research problems in which detailed radiative characterization at the small scale is crucial for the behavior and performance on the macro-scale.

\section{Acknowledgments}

We thank Martin Schneebeli from the Schweizerischen Lawinenforschungs Institut (SLF, Davos) for providing tomography data of the snow samples, and Thomas Kirchstetter and Odelle Hadley at the Lawrence Berkeley National Laboratory for providing the experimental reflectance data.

\section{References}

[1] Furler P, Scheffe J, Gorbar M, Moes L, Vogt U, Steinfeld A. Solar thermochemical CO2 splitting utilizing a reticulated porous ceria redox system. Energy Fuels 2012;26(11):7051-9.

[2] Kelzenberg MD, Boettcher SW, Petykiewicz JA, Turner-Evans DB, Putnam MC, Warren EL, et al. Enhanced absorption and carrier collection in Si wire arrays for photovoltaic applications. Nat Mater 2010;9(3):239-44.

[3] Wiscombe WJ, Warren SG. A model for the spectral albedo of snow. I: pure snow. J Atmos Sci 1980;37(12):2712-33.

[4] Warren SG, Wiscombe WJ. A model for the spectral albedo of snow. II: snow containing atmospheric aerosols. I Atmos Sci 1980;37(12):2734-45.

[5] Baillis D, Sacadura J-F, Institut. Thermal radiation properties of dispersed media: theoretical prediction and experimental characterization. J Quant Spectrosc Radiative Transfer 2000;67(5):327-63.

[6] Dombrovsky LA, Baillis D. Thermal radiation in disperse systems: an engineering approach. New York: Begell House; 2010.

[7] Mishchenko MI. Maxwell's equations, radiative transfer, and coherent backscattering: a general perspective. J Quant Spectrosc Radiative Transfer 2006;101(3):540-55.

[8] Coquard R, Baillis D. Radiative characteristics of opaque spherical paricles beds: a new method of prediction. J Thermophys Heat Transfer 2004;18(2):226-34

[9] Coquard R, Baillis D. Radiative characteristics of beds of spheres containing an absorbing and scattering medium. J Thermophys Heat Transfer 2005; 19(2):226-34.

[10] Suter S, Haussener S. Morphology engineering of porous media for enhanced solar fuel and power production. JOM 2013;65(12):1702-9.

[11] Wheeler VM, Randrianalisoa J, Tamma K, Lipiński W. Spectral radiative properties of three-dimensionally ordered macroporous ceria particles. J Quant Spectrosc Radiative Transfer 2014;143:63-72.

[12] Randrianalisoa J, Lipinski W. Effect of pore-level geometry on far-field radiative properties of three-dimensionally ordered macroporous ceria particle. Appl Opt 2014;53(7):1290-7.

[13] Haussener S, Steinfeld A. Effective heat and mass transport properties of anisotropic porous ceria for solar thermochemical fuel generation. Materials (Basel) 2012:5(1):192-209.

[14] Haussener S, Gergely M, Schneebeli M, Steinfeld A. Determination of the macroscopic optical properties of snow based on exact morphology and direct pore-level heat transfer modeling. J Geophys Res Earth Surf 2012;117(3)

[15] Randrianalisoa JH, Haussener S, Baillis D, Lipiński W. Radiative characterization of random fibrous media with optically large long cylindrical fibers. J Quant Spectrosc Radiative Transfer 2017.

[16] Coray P, Lipiński W, Steinfeld A. Experimental and numerical determination of thermal radiative properties of $\mathrm{ZnO}$ particulate media. $J$ Heat Transfer 2009;132(1):12701.

[17] Ganesan K, Dombrovsky La, Lipiński W. Visible and near-infrared optical properties of ceria ceramics. Infrared Phys Technol 2013;57:101-9.

[18] Ganesan K, Randrianalisoa J, Lipiński W. Effect of morphology on spectral radiative properties of three-dimensionally ordered macroporous ceria packed bed. J Heat Transfer 2013;135(12):122701.
[19] Stefik M, Cornuz M, Mathews N, Hisatomi T, Mhaisalkar S, Gratzel M. Transparent, conducting $\mathrm{Nb}: \mathrm{SnO} 2$ for host-guest photoelectrochemistry. Nano Lett 2012;12:5431.

[20] Furler P, Scheffe J, Marxer D, Gorbar M, Bonk A, Vogt U, et al. Thermochemical CO2 splitting via redox cycling of ceria reticulated foam structures with dual-scale porosities. Phys Chem Chem Phys 2014;16(22):10503-11.

[21] Warren SG, Brandt RE. Optical constants of ice from the ultraviolet to the microwave: a revised compilation. J Geophys Res Atmos 2008;113(14):1-10.

[22] Bohren C, Huffman D. Absorption and scattering of light by small particles. New York: John Wiley and Sons Inc.; 2004.

[23] Draine BT, Flatau PJ. Discrete-dipole approximation for scattering calculations J Opt Soc Am A 1994;11(4):1491-9.

[24] Flatau PJ, Draine BT. Fast near field calculations in the discrete dipole approximation for regular rectilinear grids. Opt Express 2012;20(2):1247-52.

[25] Frisvad JR, Christensen NJ, Jensen HW. Computing the scattering properties of participating media using Lorenz-Mie theory. ACM Trans Graphics 2007;26(3):60

[26] Purcell EM, Pennypacker CR. Scattering and absorption of light by nonspherical dielectric grains. Astrophys J 1973;186:705-14.

[27] Draine BT. The discrete-dipole approximation and its application to interstellar graphite grains. Astrophys J 1988:333:848-72.

[28] Shen Y, Draine BT, Johnson ET. Modeling porous dust grains with ballistic aggregates. I. Geometry and optical properties. Astrophys J 2008;689:260-75.

[29] Shen Y, Draine BT, Johnson ET. Modeling porous dust grains with ballistic aggregates. II. Light scattering properties. Astrophys J 2009;696(2):2126-37.

[30] Modest MF. Radiative heat transfer. Academic Press; 2013.

[31] Lipiński W, Petrasch J, Haussener S. Application of the spatial averaging theorem to radiative heat transfer in two-phase media. I Ouant Spectrosc Radiative Transfer 2010;111(1):253-8.

[32] Lipiński W, Keene D, Haussener S, Petrasch J. Continuum radiative heat transfer modeling in media consisting of optically distinct components in the limit of geometrical optics. J Quant Spectrosc Radiative Transfer 2010;111(16):2474-80.

[33] Haussener S, Lipinśki W, Petrasch J, Wyss P, Steinfeld A. Tomographic characterization of a semitransparent-particle packed bed and determination of its thermal radiative properties. J Heat Transfer 2009;131(7):72701.

[34] Tancrez M, Taine J. Direct identification of absorption and scattering coefficients and phase function of a porous medium by a Monte Carlo technique. Int J Heat Mass Transfer 2004;47(2):373-83.

[35] Petrasch J, Wyss P, Steinfeld A. Tomography-based Monte Carlo determination of radiative properties of reticulate porous ceramics. J Quant Spectrosc Radiative Transfer 2007;105(2):180-97.

[36] Solomon S. Climate change 2007-the physical science basis: working group I contribution to the fourth assessment report of the IPCC. Cambrige University Press; 2007.

[37] Xi J, Zhong B-J. Soot in diesel combustion systems. Chem Eng Technol 2006;29(6):665-73.

[38] Xu F, Faeth GM. Soot formation in laminar acetylene/air diffusion flames at atmospheric pressure. Combust Flame 2001;125(1-2):804-19.

[39] Slowik JG, Cross ES, Han J-HH, Davidovits P, Onasch TB, Jayne JT, et al. An inter-comparison of instruments measuring black carbon content of soot particles. Aerosol Sci Technol 2007:41(3):295-314.

[40] Bond TC, Bergstrom RW. Light absorption by carbonaceous particles: an investigative review. Aerosol Sci Technol 2006;40(1):27-67.

[41] Adachi K, Chung SH, Buseck PR. Shapes of soot aerosol particles and implications for their effects on climate. J Geophys Res Atmos 2010;115(15):1-9.

[42] Maricq MM, Xu N. The effective density and fractal dimension of soot particles from premixed flames and motor vehicle exhaust. J Aerosol Sc 2004:35(10):1251-74.

[43] di Stasio S, Konstandopoulos AG, Kostoglou M. Cluster-cluster aggregation kinetics and primary particle growth of soot nanoparticles in flame by light scattering and numerical simulations. I Colloid Interface Sci 2002;247(1):33-46.

[44] Jacquinet-Husson N, Crepeau L, Armante R, Boutammine C, Chédin A, Scott NA, et al. The 2009 edition of the GEISA spectroscopic database. J Quant Spectrosc Radiative Transfer 2011;112(15):2395-445.

[45] Shettle EP, Fenn RW. Models for the aerosols of the lower atmosphere and the effects of humidity variations on their optical properties; 1979. Rep. No. AFGL-TR-79-0214 p. 94.

[46] Chang H, Charalampopoulos T. Determination of the wavelength dependence of refractive indices of flame soot. Math Eng Sci 1990;430(1880).

[47] Draine BT, Flatau PJ. User guide for the discrete dipole approximation code DDSCAT.6.0. Astroph0309069 2003;1(1994):78.

[48] Johnson W, Kim S, Utegulov Z, Draine B. Surface-plasmon fields in two-dimensional arrays of gold nanodisks. Proc SPIE Vol 2008;7032 70321S-70321S-11.

[49] Scarnato BV, Vahidinia S, Richard DT, Kirchstetter TW. Effects of internal mixing and aggregate morphology on optical properties of black carbon using a discrete dipole approximation model. Atmos Chem Phys 2013:13(10):5089-101.

[50] García Fernández C, Picaud S, Devel M. Calculations of the mass absorption cross sections for carbonaceous nanoparticles modeling soot. J Quant Spectrosc Radiative Transfer 2015;164:69-81.

[51] Kahnert M. Modelling the optical and radiative properties of freshly emitted light absorbing carbon within an atmospheric chemical transport model. Atmos Chem Phys 2010;10(3):1403-16.

[52] Radney JG, You R, Ma X, Conny JM, Zachariah MR, Hodges JT, et al. Dependence of soot optical properties on particle morphology: Measurements and model comparisons. Environ Sci Technol 2014;48(6):3169-76. 
[53] Kahnert M, Devasthale A. Black carbon fractal morphology and short-wave radiative impact: a modelling study. Atmos Chem Phys 2011;11(22):11745-59.

[54] Liu L, Mishchenko MI, Patrick Arnott W. A study of radiative properties of fractal soot aggregates using the superposition T-matrix method. J Quant Spectrosc Radiative Transfer 2008;109(15):2656-63.

[55] Mishchenko MI, Travis LD, Mackowski DW. T-matrix computations of light scattering by nonspherical particles: a review. J Quant Spectrosc Radiative Transfer 1996;55(5):535-75.
[56] Hadley OL, Kirchstetter TW. Black-carbon reduction of snow albedo. Nat Clim Change 2012;2(6):437-40

[57] Flanner MG, Zender CS, Randerson JT, Rasch PJ. Present-day climate forcing and response from black carbon in snow. $J$ Geophys Res Atmos 2007; 112(11):1-17.

[58] Brandt RE, Warren SG, Clarke AD. A controlled snowmaking experiment testing the relation between black carbon content and reduction of snow albedo. Geophys Res Atmos 2011;116(8):2-7. 